\title{
A Methodology to Exploit Profit Allocation in Logistics Joint Distribution Network Optimization
}

\author{
Yong Wang, ${ }^{1}$ Xiaolei Ma, ${ }^{2}$ Maozeng Xu, ${ }^{1}$ Likun Wang, ${ }^{3}$ Yinhai Wang, ${ }^{4}$ and Yong Liu ${ }^{1}$ \\ ${ }^{1}$ School of Management, Chongqing Jiaotong University, Chongqing 400074, China \\ ${ }^{2}$ School of Transportation Science and Engineering, Beihang University, Beijing 100191, China \\ ${ }^{3}$ College of Transport \& Communications, Shanghai Maritime University, Shanghai 201306, China \\ ${ }^{4}$ Department of Civil and Environmental Engineering, University of Washington, Seattle, WA 98195-2700, USA
}

Correspondence should be addressed to Xiaolei Ma; xiaolei@buaa.edu.cn and Yinhai Wang; yinhai@uw.edu

Received 1 July 2014; Revised 9 September 2014; Accepted 26 September 2014

Academic Editor: Tsan-Ming Choi

Copyright (c) 2015 Yong Wang et al. This is an open access article distributed under the Creative Commons Attribution License, which permits unrestricted use, distribution, and reproduction in any medium, provided the original work is properly cited.

\begin{abstract}
Logistics joint distribution network (LJDN) optimization involves vehicle routes scheduling and profit allocation for multiple distribution centers. This is essentially a combinational and cooperative game optimization problem seeking to serve a number of customers with a fleet of vehicles and allocate profit among multiple centers. LJDN routing optimization based on customer clustering units can alleviate the computational complexity and improve the calculation accuracy. In addition, the profit allocation mechanism can be realized based on cooperative game theory through a negotiation procedure by the Logistics Service Provider (LSP). This paper establishes a model to minimize the total cost of the multiple centers joint distribution network when each distribution center is assigned to serve a series of distribution units. An improved particle swarm optimization (PSO) algorithm is presented to tackle the model formulation by assigning distribution centers (DCs) to distribution units. Improved PSO algorithm combines merits of PSO algorithm and genetic algorithm (GA) with global and local search capabilities. Finally, a Shapley value model based on cooperative game theory is proposed to obtain the optimal profit allocation strategy among distribution centers from nonempty coalitions. The computational results from a case study in Guiyang city, China, suggest the optimal sequential coalition of distribution centers can be achieved according to Strictly Monotonic Path (SMP).
\end{abstract}

\section{Introduction}

A logistics joint distribution network (LJDN) is usually composed of several logistics facilities (e.g., logistics centers and distribution centers) and a large number of customers [13]. Different from single-depot logistics distribution network optimization problems, the cooperation mechanism widely exists for LJDN. How to allocate the cost savings among different logistics participants in LJDN is considered as the most critical issue during the optimization procedure. Properly optimizing LJDN not only mitigates network-wide traffic congestion and reduces negative environmental effects (i.e., energy consumption and traffic pollution), but also promotes mutual cooperation for profit maximization $[4,5]$. To achieve these goals, a necessary negotiation process between multiple participants is desired, and this process can be implemented by introducing a Logistics Service Provider
(LSP) to coordinate or discussing within the existing participants [6-8]. The LSP can be defined as a Logistics Service Provider that performs corresponding logistics operations on behalf of other participants [9]. LSPs are actively looking for opportunities to increase both efficiency and profit for their own clients (i.e., participants) [10,11]. In addition, logistics companies and manufacturers tend to outsource their noncore business to a third-party for financial savings, and this raises the transportation demand and stimulates resource integration in LSP market.

LJDN optimization is a strategic and tactical procedure with multiple complicated steps, such as multiple depots (or centers) vehicle routing optimization and profit allocation procedures. Most previous studies focused on designing efficient algorithms for solving Multiple Depot Vehicle Routing Problems (MDVRP) [2, 12-14]. To ensure successful delivery 
and pickup services in MDVRP, the coordination among multiple depots should be taken prior to vehicle routing. This incurs an interesting issue on how to allocate profit within multiple logistics entities in a cooperative manner [15, 16]. Traditional MDVRP neglected such a profit allocation procedure by assuming that each logistic entity is willing to cooperate. This assumption oversimplifies the realistic condition where the logistics activities are profit-driven, and thus individual benefit should be incorporated during horizontal and vertical cooperation [11], which can be achieved via a profit allocation mechanism to retain a mutually beneficial relationship. The stability of cooperation relies on the rationality of profit allocation. Therefore, the rationality of profit allocation is the core of the logistics joint distribution network optimization [17] and should be taken into account in this study.

The cost savings from multiple depots vehicle routing optimization will be served as the input to allocate profit among various logistics entities. Therefore, developing a robust solution algorithm to find optimal solutions for MDVRP is necessary. However, in metropolitan logistics distribution network with thousands of customers, traditional approach for MDVRP may not be effective to cope with such a sophisticated scenario $[18,19]$. To alleviate the computational complexity and improve the calculation accuracy, customer clustering should be initially applied before VRP optimization. A large logistics region can be grouped into several smaller zones where customers share certain common features (i.e., geospatial location, demand, etc.). Then, customers within each zone form a clustering unit and request service from each depot. Many researchers have proposed a variety of clustering methods to study MDVRP [14, 20-24]. Thangiah and Salhi [21] presented a generalized clustering approach based on genetic algorithm, and their genetic clustering method can be further used to solve the MDVRP. Wu et al. [22] proposed a hybrid simulated annealing algorithm to solve MDVRP, where insertion and 2swap operators were used to manipulate customers from one cluster to another cluster. Dondo and Cerdá [23] developed a three-stage heuristic approach for the multiple depots routing problem with time windows and heterogeneous vehicles: cluster generation, cluster assignment and sequencing, and nodes sequencing within clusters (i.e., vehicle scheduling). Mirabi et al. [14] presented three hybrid heuristics to solve the MDVRP. Yücenur and Demirel [24] developed a genetic algorithm based on the clustering technique for studying the multidepot vehicle routing problem, and the cluster first-route second algorithm was proposed. In their paper, clustering procedures were used to group customers with similar characteristics to reduce the calculation complexity.

Customer clustering is usually an intermediate stage during the MDVRP optimization procedure. The distribution centers (DCs) can be assigned to a number of customer clustering units for delivery service, and this issue can be considered as a variant of the quadratic assignment problem (QAP) $[25,26]$. The QAP was first introduced by Koopmans and Beckmann [27] and aimed to assign $n$ facilities to $n$ locations in such a way that each facility is assigned to one exact location. The goal of the QAP is to minimize the sum of the distances multiplied by the corresponding flows and the associated cost of allocating each facility to a certain location [28-30]. Within each clustered region, the mathematical programming model and solution algorithm can be further developed to solve MDVRP $[12,15,31]$. Ho et al. [13] developed two hybrid genetic algorithms based on customer clustering techniques for dealing with MDVRP. Liu et al. [32] presented a mathematical programming model and a twophase greedy algorithm to study the full truckloads multidepot capacitated vehicle routing problem in carrier collaboration. Aras et al. [16] formulated two mixed-integer linear programming models (MILP) for selective MDVRP with pricing, and a Tabu Search on the basis of heuristic method was proposed to solve the MILP model. Bettinelli et al. [33] established an integer linear programming model and presented a branch-and-cut-and-price algorithm to solve the multidepot heterogeneous vehicle routing problem with time windows. Narasimha et al. [34] proposed an extension of antcolony technique to solve the min-max MDVRP. Tu et al. [35] presented a bi-level Voronoi diagram-based metaheuristic to tackle the large-scale MDVRP.

Based on the aforementioned discussion, a natural thought is to incorporate the profit allocation paradigm into multiple depots' vehicle routing optimization for logistics joint distribution network. However, only a small number of relevant studies have been conducted on this research domain. Özener and Ergun [36] presented cost allocation mechanisms based on the cooperative game theory, and then a set of new properties and several cost allocation schemes were proposed to study a collaborative transportation procurement network. Krajewska et al. [37] presented the profit margins resulting from horizontal cooperation among freight carriers, which is based on the cooperative game theory for a pickup and delivery problem with time windows. The possibilities of sharing these profit margins among the partners were also discussed. Wang et al. [38] constructed two mathematical models to study the optimal allocation of the module members for given garment assembly tasks in a modular production system. Frisk et al. [17] proposed a new cost allocation method based on economic models. These models include Shapley value, the nucleolus, shadow prices, and volume weights for collaborative forest transportation. Cruijssen et al. [7] proposed a novel "supplier-initiated outsourcing" procedure to exploit synergy in transportation. Lozano et al. [39] presented a linear model to allocate the cost savings among different companies when their transportation requirements are simultaneously considered. However, the above studies suffer from the following issues. (1) The network size in most studies is relatively small. When considering the large-scale logistics distribution network, customer clustering should be adopted prior to VRP optimization for reducing the calculation complexity. Consequently, the mathematical programming model and solution algorithm should be designed to optimize the logistics distribution network based on customer clustering units rather than customers. (2) Most studies focus on investigating the mechanism of profit allocation from economic perspectives but neglect the interaction between profit allocation and VRP optimization. To the best of our knowledge, no explicit architecture was developed 
to explain how optimized vehicle routings affect the profit distribution among multiple logistics participants. Therefore, a reasonable profit allocation approach based on cooperative game theory should be designed and combined with MDVRP for LJDN optimization.

This study aims to construct a multiple centers logistics joint distribution network and then develops a linear programming model with a solution algorithm to optimize the network for cost savings calculation. Based on the computed cost savings, a profit allocation approach is proposed to distribute the total profit within logistics participants and determine the optimal strategy for sequential coalitions. Compared with the previous studies, the main contributions of this paper lie in the following. (1) The model formulation is first established to minimize the total cost in the LJDN optimization procedure. (2) An improved particle swarm optimization (PSO) algorithm is proposed to assign distribution centers (DCs) to distribution units and resolve the model. (3) Shapley value model is utilized to study the profit allocation among multiple distribution centers in LJDN. (4) A real-world numerical study is undertaken to demonstrate the applicability of the proposed method.

\section{Logistics Joint Distribution Network}

Logistics joint distribution network (LJDN) can be established through negotiation. The negotiation procedure is organized by either Logistics Service Provider (LSP) or players from the distribution network [1,4,40-42]. LJDN can reasonably integrate the resources together. Therefore, it can reduce the crisscross transportation phenomenon and realize information sharing. In our study, the logistics distribution network contains multiple DCs and a large amount of customer clustering units. Figure 1 presents a logistics network structure change before and after joint distribution. DCs are independent with each other before the LJDN is established. Each customer clustering unit is a group of customers with common features, such as similar temperature controlled goods and similar geographical conditions; the customer clustering unit is referred to as a distribution unit.

The logistics distribution network structure without joint distribution exhibits a nonoptimal condition as shown in Figure 1(a), where several DCs still serve those distribution units with long distances, even if these distribution units are adjacent to other peer DCs. This is probably due to the customer loyalty and market condition $[4,5]$. For instance, customers may still continue to request the same service from their previous DCs for long-term cooperation relations. In addition, if no equivalent policies (e.g., door-to-door service, discount, etc.) are available from the other alternative DCs in the market, customers are more likely to accept services from previous DCs even if these DCs are far away from them. To optimize the unreasonable network structure, the cooperation between DCs should be promoted by reassigning distribution units to different DCs. Such a network structure adjustment may lead to cost savings compared with the previous "unreasonable" logistics network, and the generated profits are needed to distribute among multiple DCs. Therefore, the LJDN optimization becomes two critical issues on how to redesign the logistics network and then allocate the gained profits within multiple DCs in an effective manner. As shown in Figure 1(b), when LJDN is established, each distribution unit is reasonably assigned to its adjacent DC. Goods can be delivered among DCs by a fleet of semitrailer trucks, and each DC can perform a variety of routing plans to serve several distribution units with small trucks. LJDN optimization needs to consider the interplay among DCs and distribution units, and it is a multiconstraint combinatorial and game optimization issue. The goal of LJDN is to serve each customer timely and reduce the total cost of the entire system.

Under the actual circumstance, each distribution unit includes multiple customers, and the center of each distribution unit can be calculated as $\overline{x_{j}}=\sum_{o=1}^{N_{j}} x_{j o} / N_{j}, \overline{y_{j}}=$ $\sum_{o=1}^{N_{j}} y_{j o} / N_{j}$, where the coordinate for each customer $o$ in the distribution unit $j$ can be expressed as $\left(x_{j o}, y_{j o}\right)$, and the number of customers is $N_{j}$. The distance can be calculated based on each set of coordinates. For the convenience of calculation, several assumptions need to be set before LJDN is established.

(1) The flow of goods is bidirectional between DCs; this means that DCs contain both input and output flows.

(2) The customer demands are predetermined and considered relatively stable within a certain period.

(3) Semitrailer trucks are used for loading and unloading goods between the DCs, and small trucks are used for delivery between DCs and distribution units.

(4) The distribution units are served by a fleet of small trucks. Each small truck will return to its DC after serving all customers in each distribution unit. For each small truck, the total delivery distance is identical to the returning distance. It is worth noting that no vehicle routing problem (VRP) was involved to determine the sequence for both DCs and distribution units in this study.

(5) Multiple DCs are independent with each other before the LJDN is established. LJDN will be established through negotiation organized by LSP, and the ultimate optimization goal is to maximize the cost savings.

\section{Related Definitions and Model Formulation}

3.1. Related Definitions. Several related definitions are needed and described as follows.

$I\{i \mid i=1,2,3, \ldots, m\}$ denotes the set of distribution centers; in addition, $h \in I$, and $h$ represents a distribution center that is different from $i$ and denoted as $h \neq i$.

$J\{j \mid j=1,2,3, \ldots, n\}$ denotes the set of distribution units in a logistics distribution network.

$x_{i j}$ expresses the delivery quantity from the $i$ th distribution center to the $j$ th distribution unit.

$q_{j}$ denotes the demand quantity of the $j$ th distribution unit within one working period. 

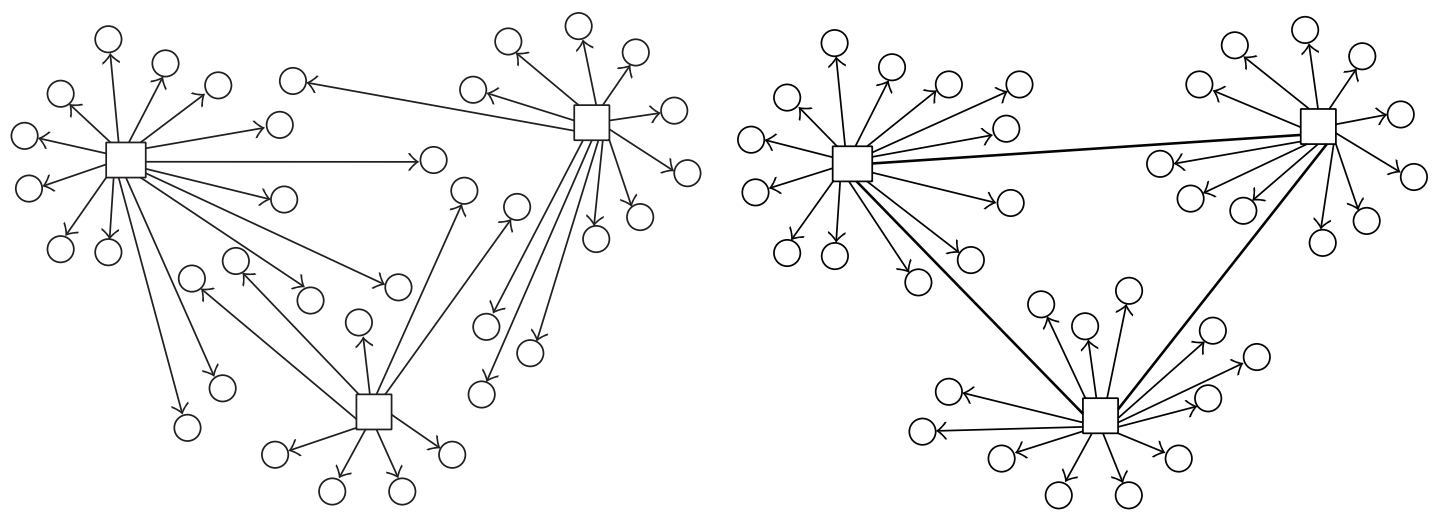

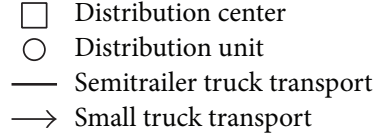

(a) Before joint distribution

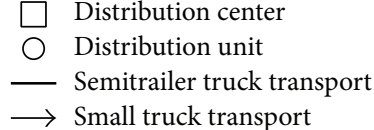

(b) After joint distribution

FIGURE 1: Logistics joint distribution network diagram.

$Q$ expresses the total demand quantity of all distribution units and can be expressed as $Q=\sum_{j} q_{j}$, where $j \in J$.

$d_{i}$ denotes the delivery capacity of the $i$ th distribution center within one working period.

$d_{i, h}$ expresses the delivery quality from distribution center $i$ to $h$.

$D$ denotes the total delivery capacity of the distribution centers and can be expressed as $D=\sum_{i} d_{i}$, where $i \in I$.

$B\left\{B_{i} \mid i=1,2, \ldots, m\right\}$ denotes the decision matrix, and $B_{i}=0$ or 1 , where $i \in I . B_{i}=1$ denotes that the distribution center $i$ agrees to cooperate in logistics joint distribution network. $B_{i}=0$ denotes that the distribution center $i$ refuses to cooperate in logistics joint distribution network.

$r_{i, j}$ denotes the distribution relation between DCs and distribution units, $r_{i, j}=0$ or 1 , where $i \in I, j \in J, r_{i, j}=$ 1 denotes that the distribution center $i$ will deliver goods to the distribution unit $j$, and $r_{i, j}=0$ indicates that the distribution center $i$ will not deliver goods to the distribution unit $j$. In addition, $\sum_{i \in I} r_{i, j}=1$.

3.2. Model Formulation. The model formulation can be considered as the objective function to minimize the total cost when each DC is assigned to serve a series of distribution units. The notations used in the logistics joint distribution network optimization formulation are listed as follows:

$\mathrm{LC}_{v}$ : loading capacity of small truck,

$\mathrm{LC}_{s}$ : loading capacity of semitrailer truck,

$C_{v}$ : fuel consumption rate of small truck (gallon/K miles),
$C_{s}$ : fuel consumption rate of semitrailer truck (gallon/K miles),

$\rho_{v}$ : gasoline price (dollar/gallon),

$\rho_{s}$ : diesel price (dollar/gallon),

$F_{v}$ : annual maintenance cost of small truck (dollar/year),

$F_{s}$ : annual maintenance cost of semitrailer truck (dollar/year),

$L_{i, h}:$ distance from the distribution center $i$ to $h$,

$L_{i, j}$ : distance from the distribution center $i$ to the distribution unit $j$,

$\lambda$ : variable cost coefficient of each distribution center,

$K$ : distance unit for fuel consumption calculation,

$T$ : number of working period,

$G_{i}$ : fixed cost of the distribution center $i$ in a working period,

$\varsigma_{i}$ : service cost (e.g., personnel cost, maintenance cost, transportation cost, etc.) of the LSP for distribution center $i$ when cooperation is achieved in a working period.

The model formulation is composed of $F_{1}, F_{2}$, and $F_{3}$; these subformulations can be shown as follows.

$F_{1}$ denotes the sum of transportation cost and annual maintenance cost for each pair of distribution centers in LJDN within a working period, and it can be calculated as

$$
F_{1}=\sum_{i, h \in I, h \neq i}\left(\frac{d_{i, h}}{\mathrm{LC}_{s}} \times \frac{C_{s}}{K} \times \rho_{s} \times L_{i, h}+\frac{d_{i, h}}{\mathrm{LC}_{s}} \times \frac{F_{s}}{T}\right) .
$$

$F_{2}$ denotes the sum of transportation cost and annual maintenance cost from each distribution center assigned to each 
distribution unit by a fleet of small trucks within a working period; it can be calculated as

$$
\begin{aligned}
F_{2}= & \sum_{i \in I} \sum_{j \in J}\left(\frac{r_{i, j} \times x_{i, j}}{\mathrm{LC}_{v}} \times \frac{C_{v}}{K} \times \rho_{v} \times 2 \times L_{i, j}\right) \\
& +\sum_{i \in I}\left(\frac{d_{i}}{\mathrm{LC}_{v}} \times \frac{F_{c}}{T}\right) .
\end{aligned}
$$

$F_{3}$ is fixed and variable costs of all distribution centers and LSP:

$$
F_{3}=\sum_{i \in I}\left(1-B_{i}\right) G_{i}+B_{i} Z_{i}+\lambda \times d_{i}
$$

The objective function for logistics joint distribution network optimization can be expressed as follows:

$$
\begin{array}{ll}
\text { Min } & F=F_{1}+F_{2}+F_{3} \\
\text { Subject to } \quad & d_{i}=\sum_{j \in J} r_{i, j} \times x_{i, j}, \quad \forall i \in I \\
& B_{i} \leq 1, \quad B_{i}=\{0,1\}, \quad \forall i \in I \\
& \sum_{j \in J} q_{j}=\sum_{i \in I} d_{i}, \quad \forall i \in I, \forall j \in J \\
& Q=D, \quad \forall i \in I, \forall j \in J \\
& \sum_{i \in I}\left(r_{i, j} \times x_{i, j}\right)=q_{j}, \quad \forall i \in I, \quad \forall j \in J \\
& \sum_{i \in I} r_{i, j}=1, \quad \forall i \in I, \forall j \in J \\
& r_{i, j}=\{0,1\}, \quad \forall i \in I, \forall j \in J .
\end{array}
$$

The objective function (4) is to minimize the total cost of a logistics joint distribution network. Constraint (5) ensures that delivery capacity is equal to total delivery quantity at distribution center $i$. Constraint (6) enumerates two scenarios that the distribution centers either agree to cooperate or not. Constraint (7) and constraint (8) guarantee that the total demand quantity should equal the total delivery quantity. Constraint (9) ensures that the total delivery quantity from all distribution centers to the distribution unit $j$ equals the demand at the distribution unit $j$. Constraint (10) assures that a distribution unit can only be served by one distribution center. In constraint (11), if the distribution center $i$ serves the distribution unit $j$, then $r_{i, j}$ is set to 1 ; otherwise, $r_{i, j}$ is set to 0 .

3.3. Shapley Value Model. When cooperation among multiple distribution centers in LJDN is achieved, each distribution unit is adequately assigned to its adjacent distribution center for VRP optimization. LSP is then required to allocate the gained profit due to network structure adjustment to each distribution center. To implement a fair and effective profit allocation strategy, the Shapley value model can be utilized in this study. The Shapley value model belongs to the cooperative game theory, which studies cooperative behavior by analyzing the negotiation process within a group of players in setting up a joint plan or contract of activities, such as profit allocation of collaboratively generated revenues. The Shapley value model is a method that presents a unique solution to the cost and profit allocation problem. Properly allocating profit can generate the synergy savings and be critical to any logistics cooperation $[17,39,43,44]$. Several related notations are needed and presented as follows:

$N$ : set of target players in a coalitional form and is called grand coalition,

$S$ : set of coalitions from the collection of all subsets of $N$,

$v(S)$ : values of all coalitions $S$,

$\phi(N, v)$ : Shapley value allocated to a certain player in the coalitions,

$T$ : subset of coalitions belonging to $S$,

$\sigma:$ the LSP's synergy requirement,

$C_{0}(i)$ : cost of player $i$ without coalition,

$C(S)$ : total cost in the $S$ by LSP,

$\Gamma$ : set of possible sequential coalitions in grand coalition $N$,

$\pi(i)$ : rank of player $i$ in sequence $\pi$,

$\eta(i, \pi, s):$ cost reduction percentages to player $i$ on step $s$ along sequence $\pi$.

When we set $N$ as a finite set of players, $2^{N}-1$ can denote the number of all subsets of $N$ excluding the null set. The elements of all subsets are called coalitions; $N$ is also known as the grand coalition. The values of all coalitions in $S$ are mapped by a characteristic function denoted by $v$. The Shapley value method is to construct a vector $\phi(N, v)$ that allocates the value $v(N)$ of the grand coalition based on the values $v(S)$ of all coalitions $S$ [45]. The Shapley value model stated in (12) expresses the profit to be allocated for player $i$ and is on the basis of the hypothesis that the grand coalition is formed by entering each player into this coalition at a time. As the player $i$ enters the coalition, player $i$ can be assigned the marginal contribution $v(S)-v(S-\{i\})$. The Shapley value is the average expected payoff of players in a completely random procedure:

$$
\phi_{i}(N, v)=\sum_{S \subset N ; i \in S} \frac{(|S|-1) !(|N|-|S|) !}{|N| !}[v(S)-v(S-\{i\})]
$$

If $S$ is assumed as the grand coalition, the subgame $(S, v)$ is given by the restriction of $v$ to $2^{S}-1$; for all $T \subset S$, the marginal 
contribution can be expressed as $v(T)-v(T-\{i\})$. The Shapley value model then becomes

$$
\phi_{i}(S, v)=\sum_{T \subset S ; i \in T} \frac{(|T|-1) !(|S|-|T|) !}{|S| !}[v(T)-v(T-\{i\})],
$$

The Shapley value model is based on four fairness properties. The calculated cost and profit allocation strategy should satisfy the four properties including efficiency, symmetry, dummy property, and additivity [7, 17, 39]. These four properties exhibit several desirable features from a practical perspective. Both the rationality and stability of allocation can be guaranteed based on these properties.

The LSP in the current logistics network is responsible to coordinate between multiple logistics entities by providing services such as warehousing, transportation management, and negotiation. Since the players in the form of coalition are served by LSP and accumulate a certain amount of profit, the LSP needs to extract a certain amount of gained profit as a result of synergy service. This share of profit is also called synergy requirement and is expressed as $\sigma \in[0,1]$. When $\sigma$ is set as a low value, the LSP will receive a lower prospected profit, and the players in LJDN are more likely to cooperate. The value $v(S)$ of a coalition $S$ in the synergy game can be determined in

$$
v(S)=(1-\sigma) \max \left\{\sum_{i \in S} C_{0}(i)-C(S), 0\right\} .
$$

In (14), $C_{0}(i)$ expresses the cost of player $i$ in the absence of synergy, while $C(S)$ is the total cost of all players served by LSP in a coalition $S$. In addition, the synergy group can be only established when the total cost of all players in the form of coalition $S$ is lower than the total cost of players without any coalition. Whenever $\sum_{i \in S} C_{0}(i)<C(S)$ happens, the players in $S$ will not accept the LSP's service, and $v(S)$ will be set to 0 .

We assume that $\Gamma$ is the set of sequences in grand coalition $N$, and these sequences contain $|N|$ ! different combinations $\pi$. $\pi(i)$ is used to express the rank of player $i$ in sequence $\pi$. The cost reduction percentages $\eta(i, \pi, s)$ can be defined as

$$
\eta(i, \pi, s)=\frac{\phi_{i}\left(\bigcup_{\pi(\mu) \leq s, \mu \in N} \mu, v\right)}{C_{0}(i)}, \quad s \geq \pi(i) .
$$

In (15), the cost reduction percentages $\eta(i, \pi, s)$ are used to explain the Strictly Monotonic Path (SMP) in the following sections [7]. SMP is considered such a sequence where the cost reduction percentages for all committed players are monotonically increasing as each player joins the coalition. The above procedure can be illustrated in Section 4.2.

\section{The Method Solving Procedure}

The method solving procedure is composed of two steps: (1) improved particle swarm optimization (PSO) algorithm is used to solve the logistics joint distribution network optimization model. The aim of this step is to assign each distribution unit into each corresponding distribution center by optimizing the total cost calculated in (4); (2) Shapley value model is then utilized to allocate the gained profit among multiple distribution centers in logistics joint distribution network, where the gained profit is calculated by comparing the costs from the optimized logistics network and the initial logistics network.

4.1. Improved PSO Algorithm Design. Particle swarm optimization (PSO) is a swarm intelligence stochastic approach. PSO is inspired by social behavior of bird flocking and optimizes the local best solution according to the particle's position and velocity $[46,47]$. PSO algorithm can be extended to solve the combinational optimization problems [4850]. This paper presents an improved PSO algorithm with crossover and mutation operations from genetic algorithm (GA). The improved PSO algorithm can increase the reliability of solving the optimization model. The relevant notations can be predefined as follows:

$v_{\gamma}^{t+1}$ : velocity of particle $\gamma$ at iteration number $t+1$, rand $(\cdot)$ : a random fraction between 0 and 1 ,

$x_{\gamma}^{t+1}$ : a position of particle $\gamma$ at iteration number $t+1$, fix $(\cdot)$ : integer the position of each particle,

$p_{\gamma}^{t}$ : individual best known position of particle $\gamma$ at iteration number $t$,

$g_{\gamma}^{t}$ : global best known position of particle $\gamma$ at iteration number $t$,

$c_{1}$ and $c_{2}$ : acceleration coefficients,

$w_{\text {int }}$ and $w_{\text {end }}$ : inertia weights,

$V$ : maximum velocity,

$T^{\prime}$ : total number of distribution centers,

rand $\operatorname{int}\left[1, T^{\prime}\right]$ : a random integer between 1 and $T^{\prime}$,

$p_{c}$ : crossover probability,

$p_{m}:$ mutation probability,

$S_{\max }:$ maximum number of iterations for improved PSO algorithm,

Swarm size: size of particle swarm.

4.1.1. Particle Encoding Scheme and Particle State Update Operations. Particle encoding scheme and evaluation function design are the key issues in the algorithm operations $[48,51-$ 55]. For the logistics joint distribution network optimization, the number of distribution centers and the location and delivery capacity of each distribution center are needed to be taken into account. Therefore, a two-dimensional particle encoding is presented in this study. The first dimension of the particles can be expressed as $1,2,3, \ldots, j, \ldots, L$, and $L$ is the total number of distribution units. The second dimension can be expressed as the sequence number of the distribution center that is assigned to serve each distribution unit, and the second dimension code will express the position of one particle. $y_{k, j}$ is the distribution center assigned to the $j$ th 
TABle 1: Two-dimensional particle encoding.

\begin{tabular}{lcclclc}
\hline Distribution unit & 1 & 2 & $\cdots$ & $j$ & $\cdots$ & $L$ \\
\hline Distribution center number & $y_{k, 1}$ & $y_{k, 2}$ & $\cdots$ & $y_{k, j}$ & $\cdots$ & $y_{k, L}$ \\
\hline
\end{tabular}

distribution unit in the $k$ th particle. For example, $y_{k, j}=1,2,3$ and $j=1,2,3, \ldots, L$. The two-dimensional particle encoding table is shown in Table 1. $y_{4,2}=3$ expresses that the third distribution center is assigned to the second distribution unit in the fourth particle.

The above encoding method ensures that each distribution unit is served by a certain distribution center in the joint distribution network. The initial population of particles can be generated by using the particle encoding method. Then, the initial fitness function value can be obtained based on the objective function, and record the initial individual best known position $(p)$ and best known fitness function value. Meanwhile, the initial global best known position $(g)$ and initial best known fitness function value also remained in the calculation procedure. To evaluate the effectiveness of improved PSO algorithm, the fitness function value should be properly defined. If the objective function is $F_{\gamma}$, the fitness function value can be shown as

$$
Z_{\gamma}=\frac{1}{F_{\gamma}} .
$$

Both the position and velocity information can be used to express the state of a particular particle. The next state of a particle depends on the current position and velocity. A particle state update mechanism is presented in the improved PSO algorithm procedure. The velocity and position can be updated through the following equations:

$$
v_{\gamma}^{t+1}=\left\{\begin{array}{cl}
w \times v_{\gamma}^{t}+c_{1} \times \operatorname{rand}(t) \times\left(p_{\gamma}^{t}-x_{\gamma}^{t}\right) & \\
+c_{2} \times \operatorname{rand}(t) \times\left(g_{\gamma}^{t}-x_{\gamma}^{t}\right) & -V \leq v_{\gamma}^{t+1} \leq V \\
-V+2 \times V \times \operatorname{rand}(t) & \text { others }
\end{array}\right.
$$$$
x_{\gamma}^{t+1}= \begin{cases}\operatorname{fix}\left(x_{\gamma}^{t}+v_{\gamma}^{t+1}\right) & -V \leq v_{\gamma}^{t+1} \leq V \\ \operatorname{rand} \operatorname{int}\left[1, T^{\prime}\right] & \text { others. }\end{cases}
$$

In addition, the inertia weight of each particle $w$ can be further described as follows:

$$
w=\frac{\left(w_{\text {int }}-w_{\text {end }}\right)\left(S_{\max }-t\right)}{S_{\max }}+w_{\text {end }} .
$$

$w$ decreases with time in (19), and $w$ can be calculated with feedback to (17) for obtaining the new velocity and position of the particle.

4.1.2. Improved PSO Algorithm Procedure. Based on the above introduction of particle encoding scheme and particle state update operations, the improved PSO algorithm is detailed as follows.

Step 1. An integer is randomly generated as each dimension of position vector in each particle within $\left[1, T^{\prime}\right]$, and the
TABLE 2: An assumptive 3-player example.

\begin{tabular}{lcccc}
\hline$S$ & $\sum_{i \in S} C_{0}(i)$ & $C(S)$ & $v(S)$ & $\phi(S, v)$ \\
\hline$\{\mathrm{A}\}$ & 200 & 160 & 40 & $(40 ; \cdot ; \cdot)$ \\
$\{\mathrm{B}\}$ & 350 & 380 & 0 & $(\cdot ; 0 ; \cdot)$ \\
$\{\mathrm{C}\}$ & 150 & 120 & 30 & $(\cdot ; \cdot ; 30)$ \\
$\{\mathrm{A}, \mathrm{B}\}$ & 550 & 510 & 40 & $(40 ; 0 ; \cdot)$ \\
$\{\mathrm{A}, \mathrm{C}\}$ & 350 & 260 & 90 & $(50 ; \cdot ; 40)$ \\
$\{\mathrm{B}, \mathrm{C}\}$ & 500 & 480 & 20 & $(\cdot ;-5 ; 25)$ \\
$\{\mathrm{A}, \mathrm{B}, \mathrm{C}\}$ & 700 & 580 & 120 & $(63 ; 8 ; 49)$ \\
\hline
\end{tabular}

initial speed vector of each particle is randomly generated within $[-V, V]$.

Step 2. Find and regenerate the unqualified particles; calculate the fitness function value $Z_{i}$ of each particle by using (16).

Step 3. The fitness function value can be used as the individual best known solution $P_{\gamma}$, and the global optimal solution $P_{g}$ can also be found.

Step 4. Execute the crossover operation based on the $p_{c}$, and calculate the fitness function of these particles; update the relevant solutions $P_{\gamma}$ and $P_{g}$.

Step 5. Execute the mutation operations based on the $p_{m}$, and calculate the fitness function of these particles; update the relevant solutions $P_{\gamma}$ and $P_{g}$.

Step 6. Calculate the new velocity and position of each particle based on (17), (18), and (19), and determine the number of iterations; if it exceeds the maximum number of iterations, the calculation procedure will be terminated, or return to Step 2.

Step 7. Calculate and select the optimal solution (i.e., position and fitness function value) from all feasible particles. This optimal solution will be the final result for the joint distribution network optimization.

In the course of above improved PSO algorithm, the particle swarm operations and genetic operations are reasonably combined. Therefore, it enhances the search space, provides a more robust global and local search capability, and improves the optimization capability of the proposed algorithm.

\subsection{Profit Allocation Application Based on Shapley Value} Model. Once the logistics joint distribution network optimization is achieved by the improved PSO algorithm, the optimal profit allocation strategy among distribution centers from nonempty coalitions can be generated. In order to understand the Shapley value model, the calculation procedure with a 3-player example is presented in Table 2 based on (13) and (14). We assume $\sigma=0$ for calculation convenience.

All of the possible coalitions are listed in Table 2, and all cost reduction percentages can be calculated and demonstrated in Figure 2. In order to establish the grand coalition, the LSP will have to select an effective cooperation strategy for profit allocation. The order of each player joining into a 


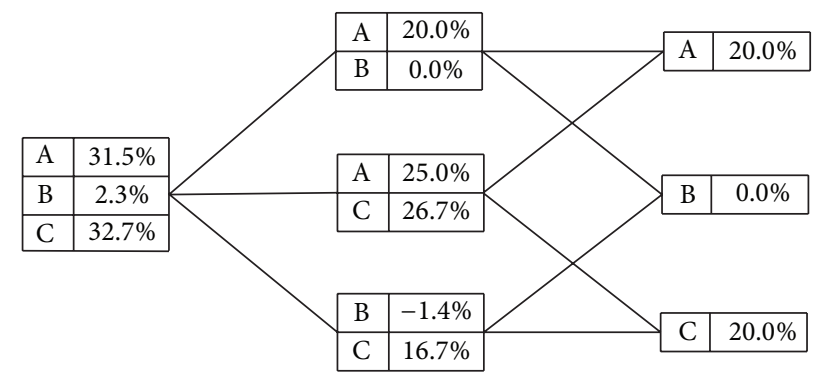

FIgURE 2: Cost reduction percentages in the 3-player example.

TABLE 3: Possible sequential coalitions for grand coalition based on SMP.

\begin{tabular}{lccccccc}
\hline \multicolumn{3}{c}{$\pi_{1}=\mathrm{ACB}$} & \multicolumn{5}{c}{$\pi_{2}=\mathrm{CAB}$} \\
\hline Player $i$ & $\mathrm{~A}$ & $\mathrm{C}$ & $\mathrm{B}$ & Player $i$ & $\mathrm{C}$ & $\mathrm{A}$ & $\mathrm{B}$ \\
\hline$\eta(i, \pi, 1)$ & $20.0 \%$ & - & - & $\eta(i, \pi, 1)$ & $20.0 \%$ & - & - \\
$\eta(i, \pi, 2)$ & $25.0 \%$ & $26.7 \%$ & - & $\eta(i, \pi, 2)$ & $26.7 \%$ & $25.0 \%$ & - \\
$\eta(i, \pi, 3)$ & $31.5 \%$ & $32.7 \%$ & $2.3 \%$ & $\eta(i, \pi, 3)$ & $32.7 \%$ & $31.5 \%$ & $2.3 \%$ \\
\hline
\end{tabular}

coalition affects the magnitude of distributed profit. Figure 2 presents the diagram for cost reduction percentage changes when each player joins a coalition. A coalition can be established through the sequence $\pi=\mathrm{ACB}$, indicating that player $\mathrm{A}$ joins a coalition, followed by player $\mathrm{C}$, and the final coalition is formed among player A, player $\mathrm{C}$, and player B. According to the definition of Strictly Monotonic Path, $\pi=\mathrm{ACB}$ and $\pi=\mathrm{CAB}$ are the only two suitable sequences satisfying the requirement of SMP, where their cost reduction percentages increase as each player joins the coalition. The next step is to find the most favorable sequence from these two SMPs as the optimal profit allocation strategy. The coalition procedures with cost reduction percentage for $\pi=$ $\mathrm{ACB}$ and $\pi=\mathrm{CAB}$ are shown in Table 3. When only one player (either player A or player $\mathrm{C}$ ) exits in the logistics network, the cost for this player A will be reduced from 200 to 160 and this player $C$ will be reduced from 150 to 120 due to the service provided by LSP, which is equivalent to a $20 \%$ reduction. However, the cost reduction rate for $\pi=\mathrm{ACB}$ when player $\mathrm{C}$ joins reaches $26.7 \%$, while this rate is only $25 \%$ when player $\mathrm{A}$ joins for $\pi=\mathrm{CAB}$. This implied that player $\mathrm{C}$ is more likely to form a coalition with player $\mathrm{A}$ because player $\mathrm{C}$ can receive a higher cost reduction compared with another scenario that player A joins the coalition with a less profit gain (e.g., 25\% cost reduction rate). Thus, the selected profit allocation strategy should be to let player A enter the logistics network, followed by player $\mathrm{C}$, and player B finally joins the coalition. This leads to the grand coalition constructed among all the players.

Without loss of generality, the above illustration can be summarized as below.

Step 1. Select the diagonal values from the cost reduction percentage matrix (e.g., Table 3 ) in possible sequential coalitions based on SMP.

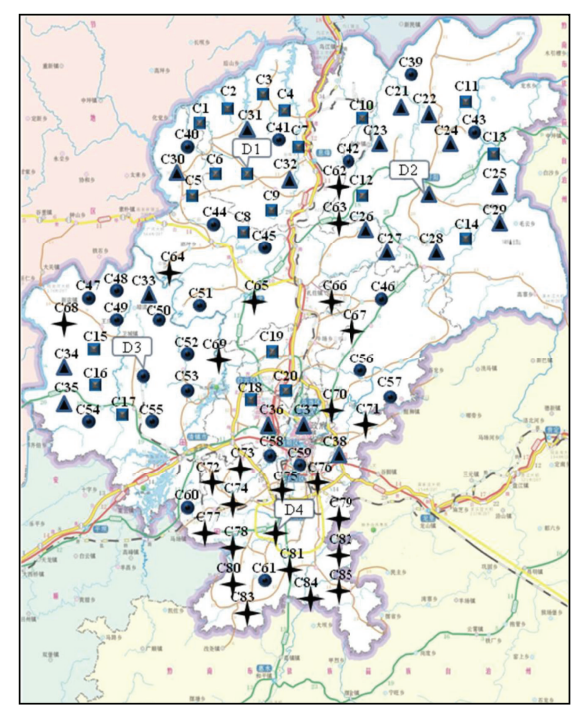

Figure 3: Distribution centers and units distribution diagram.

Step 2. Find the player with the maximum lowest cost reduction percentage in the selected diagonal values. If the cost reduction percentage remains the same for all possible sequential coalitions, then seek for another player with the maximal second lowest cost reduction rate. This process continues until at least one player can be found or all players have been searched.

Step 3. The selected sequential coalition will be considered as the candidate profit allocation strategy. If all players have been searched, select any sequential coalition as the candidate profit allocation strategy.

\section{Implementation and Analysis}

5.1. Data Source. To illustrate the applicability of the proposed methods in logistics joint distribution network optimization, a practical example in Guiyang, China, is used for the numerical study. Guiyang city is the capital of Guizhou Province and is a critical transportation hub. The locations of four DCs and 85 distribution units are demonstrated in Figure 3. 85 distribution units are, respectively, expressed as $\mathrm{C} 1, \mathrm{C} 2, \mathrm{C} 3, \ldots, \mathrm{C} 85$, and four distribution centers are, respectively, expressed as D1, D2, D3, D4. The customer units in square are assigned to D1, the customer units in triangle are assigned to D2, the circular customer units are assigned to D3, and the star-like customer units are assigned to D4. The distribution networks composed by four DCs have considerable geographical overlap shown in Figure 3, so the cooperative transportation service provided by LSP is necessary.

For the convenience of calculation, the delivery demand of each distribution unit is converted into the standard roll pallet quantity. The characteristics of four distribution centers are summarized in Table 4 including the number of customer units, periodic demand quantity (one week), and graphic symbols. 
TABLE 4: Characteristics of four distribution centers.

\begin{tabular}{lccc}
\hline DC & $\begin{array}{c}\text { Number of } \\
\text { distribution } \\
\text { units }\end{array}$ & $\begin{array}{c}\text { Periodic } \\
\text { demand } \\
\text { (roll pallets) }\end{array}$ & $\begin{array}{c}\text { Distribution } \\
\text { unit } \\
\text { symbol in } \\
\text { Figure 3 }\end{array}$ \\
\hline D1 & 20 & 32600 & 30000 \\
D2 & 18 & 39500 & \\
D3 & 23 & 41500 & \\
D4 & 24 & & \\
\hline
\end{tabular}

5.2. Improved PSO Parameter Setting and Optimization Results. In this case study, parameter settings can be determined based on previous discussion $[49,54,55]$. The parameters are given as follows.

(1) $w_{\text {int }}=0.8$ is the initial inertia weight and $w_{\text {end }}=0.3$ is the inertia weight of maximum evolution generation used for PSO speed calculation.

(2) $c_{1}=c_{2}=2$ are the acceleration coefficients used for PSO speed calculation.

(3) $V=4$ denotes the maximum velocity.

(4) $p_{c}=0.6$ and $p_{m}=0.01$ express the crossover probability and mutation probability, respectively.

(5) $S_{\max }=1000$ is the maximum number of generations.

(6) $T^{\prime}=4$ denotes the maximal random integer.

(7) Swarm size $=100$ is the number of particles used to increase the diversity of initial particle swarm.

(8) Several other parameters used in the model formulation can be set as $\lambda=1.5, \mathrm{LC}_{s}=2000, \mathrm{LC}_{v}=200$, $C_{s}=6.6, C_{v}=3.2, \rho_{v}=3.99, \rho_{s}=3.97, F_{s}=4800$, $F_{v}=1600, K=100, T=52, G_{1}=1145, G_{2}=1791$, $G_{3}=1968, G_{4}=1408, \varsigma_{1}=725, \varsigma_{2}=1166, \varsigma_{3}=1015$, and $\varsigma_{4}=1616$.

Five working days is considered one planning period, and there are $2^{4}-1$ combinations of nonempty coalitions that can be served by the LSP. The improved PSO algorithm is implemented to adequately assign each distribution unit into each distribution center by optimizing the total cost based on empirical data. The generated profit will be then redistributed among different distribution centers using the Shapley model. The optimization result over a planning period from all coalitions is shown in Table 5 .

In addition, for explanatory purposes, all distribution units affiliated with each distribution center for grand coalition are listed in Table 6.

In the next section, the cost savings due to optimized logistics joint distribution network will be allocated among different distribution centers based on Shapley value model.

5.3. Shapley Value Model Application. As previously discussed, the gained profit benefited from network optimization through LSP should be reallocated to each distribution
TABLE 5: Comparison between initial network and optimized network over a planning period.

\begin{tabular}{lccc}
\hline$S$ & Demand & $\begin{array}{c}\text { Total cost for } \\
\text { initial network }\end{array}$ & $\begin{array}{c}\text { Total cost for } \\
\text { optimized } \\
\text { network }\end{array}$ \\
\hline$\{\mathrm{D} 1\}$ & 32600 & 12639 & 12219 \\
$\{\mathrm{D} 2\}$ & 30000 & 12668 & 12043 \\
$\{\mathrm{D} 3\}$ & 39500 & 16475 & 15522 \\
$\{\mathrm{D} 4\}$ & 41500 & 15721 & 15929 \\
$\{\mathrm{D} 1, \mathrm{D} 2\}$ & 62600 & 25307 & 23024 \\
$\{\mathrm{D} 1, \mathrm{D} 3\}$ & 72100 & 29114 & 25704 \\
$\{\mathrm{D} 1, \mathrm{D} 4\}$ & 74100 & 28360 & 28136 \\
$\{\mathrm{D} 2, \mathrm{D} 3\}$ & 69500 & 29143 & 25653 \\
$\{\mathrm{D} 2, \mathrm{D} 4\}$ & 71500 & 28389 & 27737 \\
$\{\mathrm{D} 3, \mathrm{D} 4\}$ & 81000 & 32196 & 30441 \\
$\{\mathrm{D} 1, \mathrm{D} 2, \mathrm{D} 3\}$ & 102100 & 41782 & 35853 \\
$\{\mathrm{D} 1, \mathrm{D} 2, \mathrm{D} 4\}$ & 104100 & 41028 & 37914 \\
$\{\mathrm{D} 1, \mathrm{D} 3, \mathrm{D} 4\}$ & 113600 & 44835 & 40352 \\
$\{\mathrm{D} 2, \mathrm{D} 3, \mathrm{D} 4\}$ & 111000 & 44864 & 40250 \\
$\{\mathrm{D} 1, \mathrm{D} 2, \mathrm{D} 3, \mathrm{D} 4\}$ & 143600 & 57503 & 50374 \\
\hline
\end{tabular}

TABLE 6: Distribution units assignment based on grand coalition.

\begin{tabular}{cc}
\hline DC & Number of distribution units \\
\hline D1 & C1, C2, C3, C4, C5, C6, C7, C8, C9, C30, C31, C32, \\
& C40, C41, C44, C45 \\
\hline \multirow{2}{*}{ D2 } & C10, C11, C12, C13, C14, C21, C22, C23, C24, C25, \\
& C26, C27, C28, C29, C39, C42, C43, C46, C62, C63, \\
\hline D3 & C15, C16, C17, C19, C33, C34, C35, C47, C48, C49, \\
& C50, C51, C52, C53, C54, C55, C64, C65, C68, C69 \\
\hline \multirow{2}{*}{ D4 } & C18, C20, C36, C37, C38, C56, C57, C58, C59, C60, \\
& C61, C70, C71, C72, C73, C74, C75, C76, C77, C78, \\
& C79, C80, C81, C82, C83, C84, C85
\end{tabular}

center. The Shapley model can be utilized to fulfill this goal. For practical purposes, the synergy requirement is set to $\sigma=$ 0.1 in the case. The appropriate synergy requirement value reflects the negotiation power between the LSP and players from coalitions.

All of the possible coalitions have been shown in Table 7, and all cost reduction percentages and possible coalitional sequences can be calculated and are shown in Figure 4. The last column of Figure 4 shows that three coalitions have a positive percentage gain except coalition $\{\mathrm{D} 4\}$. In other words, if D1, D2, and D3 agree to accept the service provided by LSP, then, the LSP can optimize the existing logistics operations at a lower cost, compared with the scenario where each distribution center manages its own distribution units individually. In addition, the first column of Figure 4 shows that the D1, D2, D3, and D4 can obtain a certain percentage of benefits when the grand coalition is reached. This indicates that all distribution centers collaborate with the service of LSP. 


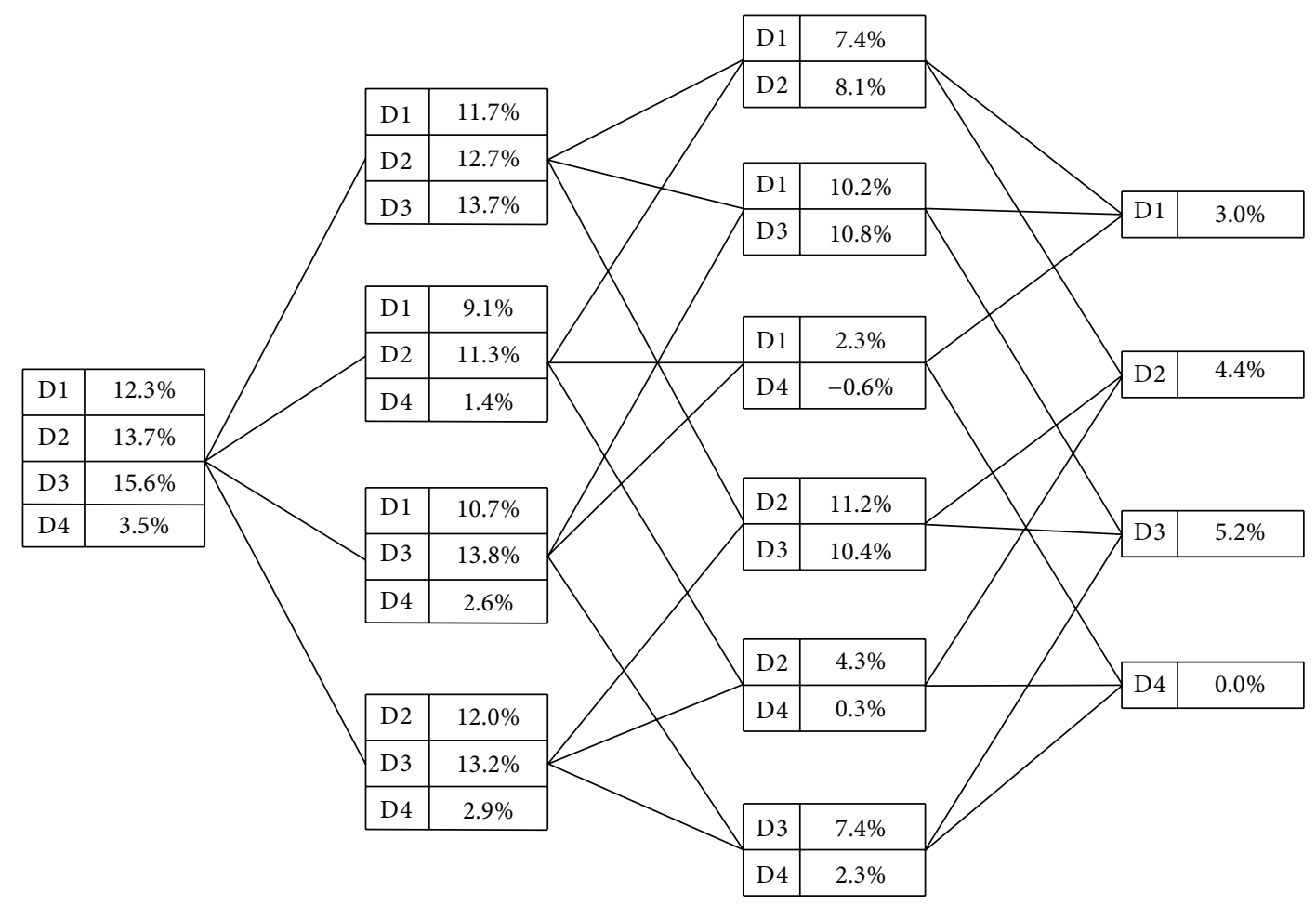

FIGURE 4: Cost reduction percentages for all coalitions.

TABLE 7: Profit allocation in joint logistics distribution network.

\begin{tabular}{|c|c|c|c|c|}
\hline$S$ & $\sum_{i \in S} C_{0}(i)$ & $C(S)$ & $v(S)$ & $\phi(S, v)$ \\
\hline$\{\mathrm{D} 1\}$ & 12639 & 12219 & 378 & $(378 ; \cdot ; \cdot ; \cdot)$ \\
\hline$\{\mathrm{D} 2\}$ & 12668 & 12043 & 563 & $(\cdot ; 563 ; \cdot ; \cdot)$ \\
\hline$\{\mathrm{D} 3\}$ & 16475 & 15522 & 858 & $(\cdot ; \cdot ; 858 ; \cdot)$ \\
\hline$\{\mathrm{D} 4\}$ & 15721 & 15929 & 0 & $(\cdot ; \cdot ; ; 0.0)$ \\
\hline$\{\mathrm{D} 1, \mathrm{D} 2\}$ & 25307 & 23024 & 2055 & $(935 ; 1020 ; \cdot ; \cdot)$ \\
\hline$\{\mathrm{D} 1, \mathrm{D} 3\}$ & 29114 & 25704 & 3069 & $(1295 ; \cdot ; 1774 ; \cdot)$ \\
\hline$\{\mathrm{D} 1, \mathrm{D} 4\}$ & 28360 & 28136 & 202 & $(290 ; \cdot ; \cdot ;-88)$ \\
\hline$\{\mathrm{D} 2, \mathrm{D} 3\}$ & 29143 & 25653 & 3141 & $(\cdot ; 1423 ; 1718 ; \cdot)$ \\
\hline$\{\mathrm{D} 2, \mathrm{D} 4\}$ & 28389 & 27737 & 587 & $(\cdot ; 545 ; \cdot ; 42)$ \\
\hline$\{\mathrm{D} 3, \mathrm{D} 4\}$ & 32196 & 30441 & 1580 & $(\cdot ; \cdot ; 1219 ; 361)$ \\
\hline$\{\mathrm{D} 1, \mathrm{D} 2, \mathrm{D} 3\}$ & 41782 & 35853 & 5336 & $(1475 ; 1603 ; 2258 ; \cdot)$ \\
\hline$\{\mathrm{D} 1, \mathrm{D} 2, \mathrm{D} 4\}$ & 41028 & 37914 & 2803 & $(1147 ; 1432 ; \cdot ; 224)$ \\
\hline$\{\mathrm{D} 1, \mathrm{D} 3, \mathrm{D} 4\}$ & 44835 & 40352 & 4035 & $(1347 ; \cdot ; 2275 ; 413)$ \\
\hline$\{\mathrm{D} 2, \mathrm{D} 3, \mathrm{D} 4\}$ & 44864 & 40250 & 4153 & $(\cdot ; 1524 ; 2168 ; 461)$ \\
\hline$\{\mathrm{D} 1, \mathrm{D} 2, \mathrm{D} 3, \mathrm{D} 4\}$ & 57503 & 50374 & 6416 & $(1558 ; 1735 ; 2578 ; 545)$ \\
\hline
\end{tabular}

5.4. Sequential Coalition Selection. Based on the discussion in Section 2, the willingness of each distribution center to join a coalition depends on how the coalition is organized. It is necessary to investigate how the sequential coalition impacts the final profit allocation. Similar to the proposed approaches in Section 4.2, we will introduce an effective approach to select the optimal sequential coalition for grand coalition establishment. The LSP will be used as the coordinator, increasing the negotiation power for all distribution centers.
Possible sequential coalitions with calculated cost reduction percentage matrices are presented in Figure 4. The sequential coalitions that satisfy the condition of SMP are further selected as shown in Tables 8-11.

The cost reduction percentages in each column from Table 8 to Table 11 are strictly monotonically increasing. This implies that these coalitions all conform to SMP provided in Section 3.3. An interesting question arises on how to select the optimal cooperation strategy from the 18 possible sequences from Tables 8-11. Based on the proposed approaches in Section 4.2, we can select $\pi=\{\mathrm{D} 1, \mathrm{D} 3, \mathrm{D} 2, \mathrm{D} 4\}$ from Table $8, \pi=\{\mathrm{D} 2, \mathrm{D} 3, \mathrm{D} 1, \mathrm{D} 4\}$ from Table $9, \pi=$ $\{\mathrm{D} 3, \mathrm{D} 2, \mathrm{D} 1, \mathrm{D} 4\}$ from Table 10 , and $\pi=\{\mathrm{D} 4, \mathrm{D} 3, \mathrm{D} 2, \mathrm{D} 1\}$ from Table 11, as the best sequential coalitions for each table. These sequential coalitions can be represented with cost reduction percentage matrices as in Table 12.

Applying the same approach in Section 4.2 into Table 12 leads to the optimal sequential coalition as $\pi_{3}=\{\mathrm{D} 3, \mathrm{D} 2$, D1, D4\}. The most feasible and beneficial cooperation strategy is described as follows. The logistics operation of D3 is initially optimized by LSP, and then a coalition is formed up between D2 and D3, followed by D1 joining the coalition. Finally, the grand coalition is established among all distribution centers. As presented in Figure 5, the cost reduction percentage is increasing for each distribution center when the coalition is updated. In reality, this strategy is favorable for both LSP and distribution centers since the high cost savings and reasonable profit allocation encourage logistics participants to cooperate with each other. 
TABLE 8: Sequential coalitions starting from D1 for grand coalition based on SMP.

\begin{tabular}{|c|c|c|c|c|c|c|c|c|c|}
\hline \multicolumn{5}{|c|}{$\pi_{1}=\{\mathrm{D} 1, \mathrm{D} 2, \mathrm{D} 3, \mathrm{D} 4\}$} & \multicolumn{5}{|c|}{$\pi_{2}=\{\mathrm{D} 1, \mathrm{D} 2, \mathrm{D} 4, \mathrm{D} 3\}$} \\
\hline Player $i$ & D1 & D2 & D3 & D4 & Player $i$ & D1 & D2 & D4 & D3 \\
\hline$\eta(i, \pi, 1)$ & $3.0 \%$ & - & - & - & $\eta(i, \pi, 1)$ & $3.0 \%$ & - & - & - \\
\hline$\eta(i, \pi, 2)$ & $7.4 \%$ & $8.1 \%$ & - & - & $\eta(i, \pi, 2)$ & $7.4 \%$ & $8.1 \%$ & - & - \\
\hline$\eta(i, \pi, 3)$ & $11.7 \%$ & $12.7 \%$ & $13.7 \%$ & - & $\eta(i, \pi, 3)$ & $9.1 \%$ & $11.3 \%$ & $1.4 \%$ & - \\
\hline$\eta(i, \pi, 4)$ & $12.3 \%$ & $13.7 \%$ & $15.6 \%$ & $3.5 \%$ & $\eta(i, \pi, 4)$ & $12.3 \%$ & $13.7 \%$ & $3.5 \%$ & $15.6 \%$ \\
\hline \multicolumn{5}{|c|}{$\pi_{3}=\{\mathrm{D} 1, \mathrm{D} 3, \mathrm{D} 2, \mathrm{D} 4\}$} & \multicolumn{5}{|c|}{$\pi_{4}=\{\mathrm{D} 1, \mathrm{D} 3, \mathrm{D} 4, \mathrm{D} 2\}$} \\
\hline Player $i$ & D1 & D3 & D2 & D4 & Player $i$ & D1 & D3 & $\mathrm{D} 4$ & D2 \\
\hline$\eta(i, \pi, 1)$ & $3.0 \%$ & - & - & - & $\eta(i, \pi, 1)$ & $3.0 \%$ & - & - & - \\
\hline$\eta(i, \pi, 2)$ & $10.2 \%$ & $10.8 \%$ & - & - & $\eta(i, \pi, 2)$ & $10.2 \%$ & $10.8 \%$ & - & - \\
\hline$\eta(i, \pi, 3)$ & $11.7 \%$ & $13.7 \%$ & $12.7 \%$ & - & $\eta(i, \pi, 3)$ & $10.7 \%$ & $13.8 \%$ & $2.6 \%$ & - \\
\hline$\eta(i, \pi, 4)$ & $12.3 \%$ & $15.6 \%$ & $13.7 \%$ & $3.5 \%$ & $\eta(i, \pi, 4)$ & $12.3 \%$ & $15.6 \%$ & $3.5 \%$ & $13.7 \%$ \\
\hline
\end{tabular}

TABLE 9: Sequential coalitions starting from D2 for grand coalition based on SMP.

\begin{tabular}{|c|c|c|c|c|c|c|c|c|c|}
\hline \multicolumn{4}{|c|}{$\pi_{1}=\{\mathrm{D} 2, \mathrm{D} 1, \mathrm{D} 3, \mathrm{D} 4\}$} & & \multicolumn{5}{|c|}{$\pi_{2}=\{\mathrm{D} 2, \mathrm{D} 1, \mathrm{D} 4, \mathrm{D} 3\}$} \\
\hline Player $i$ & D2 & D1 & D3 & D4 & Player $i$ & D2 & D1 & D4 & D3 \\
\hline$\eta(i, \pi, 1)$ & $4.4 \%$ & - & - & - & $\eta(i, \pi, 1)$ & $4.4 \%$ & - & - & - \\
\hline$\eta(i, \pi, 2)$ & $8.1 \%$ & $7.4 \%$ & - & - & $\eta(i, \pi, 2)$ & $8.1 \%$ & $7.4 \%$ & - & - \\
\hline$\eta(i, \pi, 3)$ & $12.7 \%$ & $11.7 \%$ & $13.7 \%$ & - & $\eta(i, \pi, 3)$ & $11.3 \%$ & $9.1 \%$ & $1.4 \%$ & - \\
\hline$\eta(i, \pi, 4)$ & $13.7 \%$ & $12.3 \%$ & $15.6 \%$ & $3.5 \%$ & $\eta(i, \pi, 4)$ & $13.7 \%$ & $12.3 \%$ & $3.5 \%$ & $15.6 \%$ \\
\hline \multicolumn{4}{|c|}{$\pi_{3}=\{\mathrm{D} 2, \mathrm{D} 3, \mathrm{D} 1, \mathrm{D} 4\}$} & & \multicolumn{5}{|c|}{$\pi_{4}=\{\mathrm{D} 2, \mathrm{D} 3, \mathrm{D} 4, \mathrm{D} 1\}$} \\
\hline Player $i$ & D2 & D3 & D1 & $\mathrm{D} 4$ & Player $i$ & D2 & D3 & $\mathrm{D} 4$ & D1 \\
\hline$\eta(i, \pi, 1)$ & $4.4 \%$ & - & - & - & $\eta(i, \pi, 1)$ & $4.4 \%$ & - & - & - \\
\hline$\eta(i, \pi, 2)$ & $11.2 \%$ & $10.4 \%$ & - & - & $\eta(i, \pi, 2)$ & $11.2 \%$ & $10.4 \%$ & - & - \\
\hline$\eta(i, \pi, 3)$ & $12.7 \%$ & $13.7 \%$ & $11.7 \%$ & - & $\eta(i, \pi, 3)$ & $12.0 \%$ & $13.2 \%$ & $2.9 \%$ & - \\
\hline$\eta(i, \pi, 4)$ & $13.7 \%$ & $15.6 \%$ & $12.3 \%$ & $3.5 \%$ & $\eta(i, \pi, 4)$ & $13.7 \%$ & $15.6 \%$ & $3.5 \%$ & $12.3 \%$ \\
\hline
\end{tabular}

TABLE 10: Sequential coalitions starting from D3 for grand coalition based on SMP.

\begin{tabular}{|c|c|c|c|c|c|c|c|c|c|}
\hline \multicolumn{5}{|c|}{$\pi_{1}=\{\mathrm{D} 3, \mathrm{D} 1, \mathrm{D} 2, \mathrm{D} 4\}$} & \multicolumn{5}{|c|}{$\pi_{2}=\{\mathrm{D} 3, \mathrm{D} 1, \mathrm{D} 4, \mathrm{D} 2\}$} \\
\hline Player $i$ & D3 & D1 & D2 & D4 & Player $i$ & D3 & D1 & D4 & D2 \\
\hline$\eta(i, \pi, 1)$ & $5.2 \%$ & - & - & - & $\eta(i, \pi, 1)$ & $5.2 \%$ & - & - & - \\
\hline$\eta(i, \pi, 2)$ & $10.8 \%$ & $10.2 \%$ & - & - & $\eta(i, \pi, 2)$ & $10.8 \%$ & $10.2 \%$ & - & - \\
\hline$\eta(i, \pi, 3)$ & $13.7 \%$ & $11.7 \%$ & $12.7 \%$ & - & $\eta(i, \pi, 3)$ & $13.8 \%$ & $10.7 \%$ & $2.6 \%$ & - \\
\hline$\eta(i, \pi, 4)$ & $15.6 \%$ & $12.3 \%$ & $13.7 \%$ & $3.5 \%$ & $\eta(i, \pi, 4)$ & $15.6 \%$ & $12.3 \%$ & $3.5 \%$ & $13.7 \%$ \\
\hline \multicolumn{5}{|c|}{$\pi_{3}=\{\mathrm{D} 3, \mathrm{D} 2, \mathrm{D} 1, \mathrm{D} 4\}$} & \multicolumn{5}{|c|}{$\pi_{4}=\{\mathrm{D} 3, \mathrm{D} 2, \mathrm{D} 4, \mathrm{D} 1\}$} \\
\hline Player $i$ & D3 & D2 & D1 & $\mathrm{D} 4$ & Player $i$ & D3 & D2 & D4 & D1 \\
\hline$\eta(i, \pi, 1)$ & $5.2 \%$ & - & - & - & $\eta(i, \pi, 1)$ & $5.2 \%$ & - & - & - \\
\hline$\eta(i, \pi, 2)$ & $10.4 \%$ & $11.2 \%$ & - & - & $\eta(i, \pi, 2)$ & $10.4 \%$ & $11.2 \%$ & - & - \\
\hline$\eta(i, \pi, 3)$ & $13.7 \%$ & $12.7 \%$ & $11.7 \%$ & - & $\eta(i, \pi, 3)$ & $13.2 \%$ & $12.0 \%$ & $2.9 \%$ & - \\
\hline$\eta(i, \pi, 4)$ & $15.6 \%$ & $13.7 \%$ & $12.3 \%$ & $3.5 \%$ & $\eta(i, \pi, 4)$ & $15.6 \%$ & $13.7 \%$ & $3.5 \%$ & $12.3 \%$ \\
\hline \multicolumn{5}{|c|}{$\pi_{5}=\{\mathrm{D} 3, \mathrm{D} 4, \mathrm{D} 1, \mathrm{D} 2\}$} & \multicolumn{5}{|c|}{$\pi_{6}=\{\mathrm{D} 3, \mathrm{D} 4, \mathrm{D} 2, \mathrm{D} 1\}$} \\
\hline Player $i$ & D3 & D4 & D1 & D2 & Player $i$ & D3 & D4 & $\mathrm{D} 2$ & D1 \\
\hline$\eta(i, \pi, 1)$ & $5.2 \%$ & - & - & - & $\eta(i, \pi, 1)$ & $5.2 \%$ & - & - & - \\
\hline$\eta(i, \pi, 2)$ & $7.4 \%$ & $2.3 \%$ & - & - & $\eta(i, \pi, 2)$ & $7.4 \%$ & $2.3 \%$ & - & - \\
\hline$\eta(i, \pi, 3)$ & $13.8 \%$ & $2.6 \%$ & $10.7 \%$ & - & $\eta(i, \pi, 3)$ & $13.2 \%$ & $2.9 \%$ & $12.0 \%$ & - \\
\hline$\eta(i, \pi, 4)$ & $15.6 \%$ & $3.5 \%$ & $12.3 \%$ & $13.7 \%$ & $\eta(i, \pi, 4)$ & $15.6 \%$ & $3.5 \%$ & $13.7 \%$ & $12.3 \%$ \\
\hline
\end{tabular}


TABLE 11: Sequential coalitions starting from D4 for grand coalition based on SMP.

\begin{tabular}{|c|c|c|c|c|c|c|c|c|c|}
\hline \multicolumn{5}{|c|}{$\pi_{1}=\{\mathrm{D} 4, \mathrm{D} 2, \mathrm{D} 1, \mathrm{D} 3\}$} & \multicolumn{5}{|c|}{$\pi_{2}=\{\mathrm{D} 4, \mathrm{D} 2, \mathrm{D} 3, \mathrm{D} 1\}$} \\
\hline Player $i$ & D4 & D2 & D1 & D3 & Player $i$ & D4 & D2 & D3 & D1 \\
\hline$\eta(i, \pi, 1)$ & $0.0 \%$ & - & - & - & $\eta(i, \pi, 1)$ & $0.0 \%$ & - & - & - \\
\hline$\eta(i, \pi, 2)$ & $0.3 \%$ & $4.3 \%$ & - & - & $\eta(i, \pi, 2)$ & $0.3 \%$ & $4.3 \%$ & - & - \\
\hline$\eta(i, \pi, 3)$ & $1.4 \%$ & $11.3 \%$ & $9.1 \%$ & - & $\eta(i, \pi, 3)$ & $2.9 \%$ & $12.0 \%$ & $13.2 \%$ & - \\
\hline$\eta(i, \pi, 4)$ & $3.5 \%$ & $13.7 \%$ & $12.3 \%$ & $15.6 \%$ & $\eta(i, \pi, 4)$ & $3.5 \%$ & $13.7 \%$ & $15.6 \%$ & $12.3 \%$ \\
\hline \multicolumn{5}{|c|}{$\pi_{3}=\{\mathrm{D} 4, \mathrm{D} 3, \mathrm{D} 1, \mathrm{D} 2\}$} & \multicolumn{5}{|c|}{$\pi_{4}=\{\mathrm{D} 4, \mathrm{D} 3, \mathrm{D} 2, \mathrm{D} 1\}$} \\
\hline Player $i$ & D4 & D3 & D1 & D2 & Player $i$ & D4 & D3 & D2 & D1 \\
\hline$\eta(i, \pi, 1)$ & $0.0 \%$ & - & - & - & $\eta(i, \pi, 1)$ & $0.0 \%$ & - & - & - \\
\hline$\eta(i, \pi, 2)$ & $2.3 \%$ & $7.4 \%$ & - & - & $\eta(i, \pi, 2)$ & $2.3 \%$ & $7.4 \%$ & - & - \\
\hline$\eta(i, \pi, 3)$ & $2.6 \%$ & $13.8 \%$ & $10.7 \%$ & - & $\eta(i, \pi, 3)$ & $2.9 \%$ & $13.2 \%$ & $12.0 \%$ & - \\
\hline$\eta(i, \pi, 4)$ & $3.5 \%$ & $15.6 \%$ & $12.3 \%$ & $13.7 \%$ & $\eta(i, \pi, 4)$ & $3.5 \%$ & $15.6 \%$ & $13.7 \%$ & $12.3 \%$ \\
\hline
\end{tabular}

TABLE 12: Possible sequential coalitions for grand coalition based on SMP.

\begin{tabular}{|c|c|c|c|c|c|c|c|c|c|}
\hline \multicolumn{5}{|c|}{$\pi_{1}=\{\mathrm{D} 1, \mathrm{D} 3, \mathrm{D} 2, \mathrm{D} 4\}$} & \multicolumn{5}{|c|}{$\pi_{2}=\{\mathrm{D} 2, \mathrm{D} 3, \mathrm{D} 1, \mathrm{D} 4\}$} \\
\hline Player $i$ & D1 & D3 & $\mathrm{D} 2$ & D4 & Player $i$ & D2 & D3 & D1 & D4 \\
\hline$\eta(i, \pi, 1)$ & $3.0 \%$ & - & - & - & $\eta(i, \pi, 1)$ & $4.4 \%$ & - & - & - \\
\hline$\eta(i, \pi, 2)$ & $10.2 \%$ & $10.8 \%$ & - & - & $\eta(i, \pi, 2)$ & $11.2 \%$ & $10.4 \%$ & - & - \\
\hline$\eta(i, \pi, 3)$ & $11.7 \%$ & $13.7 \%$ & $12.7 \%$ & - & $\eta(i, \pi, 3)$ & $12.7 \%$ & $13.7 \%$ & $11.7 \%$ & - \\
\hline$\eta(i, \pi, 4)$ & $12.3 \%$ & $15.6 \%$ & $13.7 \%$ & $3.5 \%$ & $\eta(i, \pi, 4)$ & $13.7 \%$ & $15.6 \%$ & $12.3 \%$ & $3.5 \%$ \\
\hline \multicolumn{4}{|c|}{$\pi_{3}=\{\mathrm{D} 3, \mathrm{D} 2, \mathrm{D} 1, \mathrm{D} 4\}$} & & \multicolumn{5}{|c|}{$\pi_{4}=\{\mathrm{D} 4, \mathrm{D} 3, \mathrm{D} 2, \mathrm{D} 1\}$} \\
\hline Player $i$ & D3 & D2 & D1 & D4 & Player $i$ & D4 & D3 & D2 & D1 \\
\hline$\eta(i, \pi, 1)$ & $5.2 \%$ & - & - & - & $\eta(i, \pi, 1)$ & $0.0 \%$ & - & - & - \\
\hline$\eta(i, \pi, 2)$ & $10.4 \%$ & $11.2 \%$ & - & - & $\eta(i, \pi, 2)$ & $2.3 \%$ & $7.4 \%$ & - & - \\
\hline$\eta(i, \pi, 3)$ & $13.7 \%$ & $12.7 \%$ & $11.7 \%$ & - & $\eta(i, \pi, 3)$ & $2.9 \%$ & $13.2 \%$ & $12.0 \%$ & - \\
\hline$\eta(i, \pi, 4)$ & $15.6 \%$ & $13.7 \%$ & $12.3 \%$ & $3.5 \%$ & $\eta(i, \pi, 4)$ & $3.5 \%$ & $15.6 \%$ & $13.7 \%$ & $12.3 \%$ \\
\hline
\end{tabular}

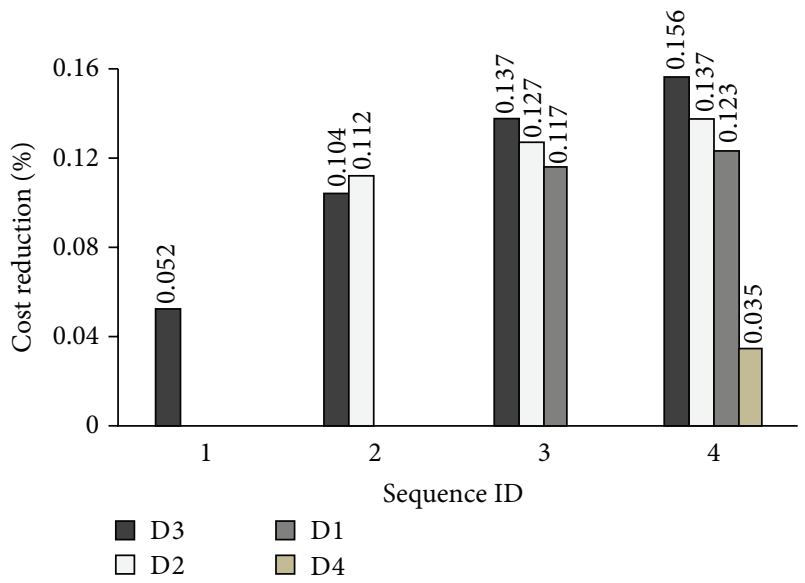

FIGURE 5: The cost reduction percentage diagram for the optimal sequential coalition.

The LSP receives $10 \%$ of the total savings (i.e., 713 USD per week) since the synergy requirement is set to $\sigma=0.1$. The remaining profit is required to distribute among D1, D2, D3, and D4 by following the Shapley value model. The distribution center D3 receives the highest cost reduction percentage due to the high customer demand and unoptimized logistics network structure. Nevertheless, the cost reduction percentage for distribution center D4 is lowest. This is probably because the surrounding traffic as well as economic condition is ideal and thus leaves little room to improve the existing logistics optimization plan. Moreover, the LSP provides a higher service cost for D4 compared with other distribution centers. Consequently, the gained profit for D4 is reduced.

The synergy requirement $\sigma$ varies depending on each distribution center's negotiation capability. LSP can decrease its synergy requirement for a certain distribution center if this distribution center is able to negotiate with other participants independently. Similarly, it is possible that the LSP may not able to persuade a certain distribution center (e.g., D4) to join a coalition when a specific synergy requirement is provided. In this case, the grand coalition will downgrade to $\{\mathrm{D} 1, \mathrm{D} 2, \mathrm{D} 3\}$, and the optimal sequential coalition $\pi=$ $\{\mathrm{D} 3, \mathrm{D} 2, \mathrm{D} 1\}$ can be generated based on the similar calculation procedure in Section 4.2. If no distribution center is willing to cooperate, the LSP should reconsider its synergy plan by lowering the synergy requirement.

\section{Conclusions}

This paper studies the logistics joint distribution network optimization problem, where multiple DCs and customers exist and interact with each other. The LJDN can be 
constructed by either LSP or existing DCs in the logistics network. A novel approach is proposed to optimize the joint logistics network and allocate the gained profit among DCs. The Logistics Service Provider (LSP) bridges both the collaborative network optimization problem and profit allocation problem and distributes the cost savings to each DC from the nonempty coalition. This further reduces the complexity and enhances the robustness of designing a largescale logistics network.

A joint distribution model formulation is initially built to optimize the total cost of nonempty coalition logistics systems. This model uses an improved PSO algorithm, and then a Shapley value model is utilized to perform the profit allocation among DCs from nonempty coalitions. Finally, the optimal sequential coalition can be obtained according to Strictly Monotonic Path (SMP) theory-all DCs can receive benefits when each DC joins the coalition. To evaluate the effectiveness of the LJDN optimization methods, a computational experiment in Guiyang city, China, was conducted. By properly adjusting the synergy requirement value $\sigma$, the results can be used to optimize the logistics distribution network and determine the optimal profit allocation strategy in a cooperative and effective fashion.

An interesting direction for further research is to optimize the multilevel logistics distribution network (MLLDN). In addition, multiple LSPs coexist in MLLDN, where each DC may be competitively served by several LSPs. In this case, the new profit allocation model needs to be established by reconsidering the ownership of each DC. To prevent a certain LSP from dominating the market, the heterogeneity of synergy requirement values should be incorporated to improve the profit allocation model.

\section{Conflict of Interests}

The authors declare that there is no conflict of interests regarding to the publication of this paper.

\section{Acknowledgments}

This research is supported by National Natural Science Foundation of China (Project nos. 71402011, 71471024, and 51408019), National Social Science Foundation of Chongqing of China (no. 2013YBJJ035), and the Scientific and Technological Research Program of Chongqing Municipal Education Commission (no. KJ1400307). Special thanks are given to Matt Dunlap from University of Washington, USA, for his valuable suggestions and revisions. In addition, helpful comments from anonymous reviewers are appreciated.

\section{References}

[1] C.-I. Hsu and W.-T. Chen, "Optimizing fleet size and delivery scheduling for multi-temperature food distribution," Applied Mathematical Modelling, vol. 38, no. 3, pp. 1077-1091, 2014.

[2] Y. Wang, X.-L. Ma, Y.-H. Wang, H.-J. Mao, and Y. Zhang, "Location optimization of multiple distribution centers under fuzzy environment," Journal of Zhejiang University: Science A, vol. 13, no. 10, pp. 782-798, 2012.
[3] Y. Wang, X. Ma, and Y. Lao, "A fuzzy-based customer clustering approach with hierarchical structure for logistics network optimization," Expert Systems with Applications, vol. 41, no. 2, pp. 521-534, 2014.

[4] B. C. Cha, I. K. Moon, and J. H. Park, "The joint replenishment and delivery scheduling of the one-warehouse, nretailer system," Transportation Research Part E: Logistics and Transportation Review, vol. 44, no. 5, pp. 720-730, 2008.

[5] O. Wangapisit, E. Taniguchi, J. S. E. Teo, and A. G. Qureshi, "Multi-agent systems modeling for evaluating joint delivery systems," Procedia-Social and Behavioral Sciences, vol. 125, pp. 472-483, 2014.

[6] M. Nagarajan and G. Sošić, "Game-theoretic analysis of cooperation among supply chain agents: review and extensions," European Journal of Operational Research, vol. 187, no. 3, pp. 719-745, 2008.

[7] F. Cruijssen, P. Borm, H. Fleuren, and H. Hamers, "Supplierinitiated outsourcing: a methodology to exploit synergy in transportation," European Journal of Operational Research, vol. 207, no. 2, pp. 763-774, 2010.

[8] N. Saeed, "Cooperation among freight forwarders: mode choice and intermodal freight transport," Research in Transportation Economics, vol. 42, no. 1, pp. 77-86, 2013.

[9] R. Rajesh, S. Pugazhendhi, K. Ganesh, Y. Ducq, and S. C. Lenny Koh, "Generic balanced scorecard framework for third party logistics service provider," International Journal of Production Economics, vol. 140, no. 1, pp. 269-282, 2012.

[10] V. Carbone and M. A. Stone, "Growth and relational strategies used by the European logistics service providers: rationale and outcomes," Transportation Research Part E: Logistics and Transportation Review, vol. 41, no. 6, pp. 495-510, 2005.

[11] F. Cruijssen, M. Cools, and W. Dullaert, "Horizontal cooperation in logistics: opportunities and impediments," Transportation Research Part E: Logistics and Transportation Review, vol. 43, no. 2, pp. 129-142, 2007.

[12] G. Nagy and S. Salhi, "Heuristic algorithms for single and multiple depot vehicle routing problems with pickups and deliveries," European Journal of Operational Research, vol. 162, no. 1, pp. 126-141, 2005.

[13] W. Ho, G. T. S. Ho, P. Ji, and H. C. W. Lau, "A hybrid genetic algorithm for the multi-depot vehicle routing problem," Engineering Applications of Artificial Intelligence, vol. 21, no. 4, pp. 548-557, 2008.

[14] M. Mirabi, S. M. T. Fatemi Ghomi, and F. Jolai, "Efficient stochastic hybrid heuristics for the multi-depot vehicle routing problem," Robotics and Computer-Integrated Manufacturing, vol. 26, no. 6, pp. 564-569, 2010.

[15] B. Crevier, J.-F. Cordeau, and G. Laporte, "The multi-depot vehicle routing problem with inter-depot routes," European Journal of Operational Research, vol. 176, no. 2, pp. 756-773, 2007.

[16] N. Aras, D. Aksen, and M. T. Tekin, "Selective multi-depot vehicle routing problem with pricing," Transportation Research Part C: Emerging Technologies, vol. 19, no. 5, pp. 866-884, 2011.

[17] M. Frisk, M. Göthe-Lundgren, K. Jörnsten, and M. Rönnqvist, "Cost allocation in collaborative forest transportation," European Journal of Operational Research, vol. 205, no. 2, pp. 448458, 2010.

[18] Z. Wang, Q. H. Zhang, B. Yang, and W. W. He, "4/R/I/T distribution logistics network 0-1 programming model and application," Computers \& Industrial Engineering, vol. 35, no. 2, pp. 265-378, 2008. 
[19] M. Qi, W.-H. Lin, N. Li, and L. Miao, "A spatiotemporal partitioning approach for large-scale vehicle routing problems with time windows," Transportation Research. Part E: Logistics and Transportation Review, vol. 48, no. 1, pp. 248-257, 2012.

[20] S. Salhi and G. Nagy, "A cluster insertion heuristic for single and multiple depot vehicle routing problems with backhauling," Journal of the Operational Research Society, vol. 50, no. 10, pp. 1034-1042, 1999.

[21] S. R. Thangiah and S. Salhi, "Genetic clustering: an adaptive heuristic for the multidepot vehicle routing problem," Applied Artificial Intelligence, vol. 15, no. 4, pp. 361-383, 2001.

[22] T.-H. Wu, C. Low, and J.-W. Bai, "Heuristic solutions to multidepot location-routing problems," Computers and Operations Research, vol. 29, no. 10, pp. 1393-1415, 2002.

[23] R. Dondo and J. Cerdá, "A cluster-based optimization approach for the multi-depot heterogeneous fleet vehicle routing problem with time windows," European Journal of Operational Research, vol. 176, no. 3, pp. 1478-1507, 2007.

[24] G. N. Yücenur and N. Ç. Demirel, "A new geometric shapebased genetic clustering algorithm for the multi-depot vehicle routing problem," Expert Systems with Applications, vol. 38, no. 9, pp. 11859-11865, 2011.

[25] G. Maquera, M. Laguna, D. A. Gandelman, and A. P. Sant'Anna, "Scatter search applied to the vehicle routing problem with simultaneous delivery and pickup," International Journal of Applied Metaheuristic Computing, vol. 2, no. 2, pp. 1-20, 2011.

[26] T. James, C. Rego, and F. Glover, "Multistart tabu search and diversification strategies for the quadratic assignment problem," IEEE Transactions on Systems, Man, and Cybernetics Part A:Systems and Humans, vol. 39, no. 3, pp. 579-596, 2009.

[27] T. C. Koopmans and M. Beckmann, "Assignment problems and the location of economic activities," Econometrica, vol. 25, pp. 53-76, 1957.

[28] H. Zhang, C. Beltran-Royo, and M. Constantino, "Effective formulation reductions for the quadratic assignment problem," Computers and Operations Research, vol. 37, no. 11, pp. 20072016, 2010.

[29] C. Özkale and A. Fığlalı, "Evaluation of the multiobjective ant colony algorithm performances on biobjective quadratic assignment problems," Applied Mathematical Modelling, vol. 37, no. 14-15, pp. 7822-7838, 2013.

[30] M. S. Hussin and T. Stützle, "Tabu search vs. simulated annealing as a function of the size of quadratic assignment problem instances," Computers and Operations Research, vol. 43, pp. 286291, 2014.

[31] D. Gulczynski, B. Golden, and E. Wasil, “The multi-depot split delivery vehicle routing problem: an integer programmingbased heuristic, new test problems, and computational results," Computers and Industrial Engineering, vol. 61, no. 3, pp. 794804, 2011.

[32] R. Liu, Z. Jiang, R. Y. K. Fung, F. Chen, and X. Liu, “Two-phase heuristic algorithms for full truckloads multi-depot capacitated vehicle routing problem in carrier collaboration," Computers and Operations Research, vol. 37, no. 5, pp. 950-959, 2010.

[33] A. Bettinelli, A. Ceselli, and G. Righini, "A branch-and-cutand-price algorithm for the multi-depot heterogeneous vehicle routing problem with time windows," Transportation Research C: Emerging Technologies, vol. 19, no. 5, pp. 723-740, 2011.

[34] K. V. Narasimha, E. Kivelevitch, B. Sharma, and M. Kumar, "An ant colony optimization technique for solving min-max multi-depot vehicle routing problem," Swarm and Evolutionary Computation, vol. 13, pp. 63-73, 2013.
[35] W. Tu, Z. Fang, Q. Li, S.-L. Shaw, and B. Chen, "A bi-level Voronoi diagram-based metaheuristic for a large-scale multidepot vehicle routing problem," Transportation Research Part E: Logistics and Transportation Review, vol. 61, pp. 84-97, 2014.

[36] O. Ö. Özener and Ö. Ergun, "Allocating costs in a collaborative transportation procurement network," Transportation Science, vol. 42, no. 2, pp. 146-165, 2008.

[37] M. A. Krajewska, H. Kopfer, G. Laporte, S. Ropke, and G. Zaccour, "Horizontal cooperation among freight carriers: request allocation and profit sharing," Journal of the Operational Research Society, vol. 59, no. 11, pp. 1483-1491, 2008.

[38] X. Wang, C.-H. Chiu, and W. Guo, "Improving the performance of modular production in the apparel assembly: a mathematical programming approach," Mathematical Problems in Engineering, vol. 2014, Article ID 472781, 7 pages, 2014.

[39] S. Lozano, P. Moreno, B. Adenso-Díaz, and E. Algaba, "Cooperative game theory approach to allocating benefits of horizontal cooperation," European Journal of Operational Research, vol. 229, no. 2, pp. 444-452, 2013.

[40] X. D. Lin, L. J. Ma, and Z. X. Zheng, "Customized transportation, equity participation and cooperation performance within logistics supply chains," Mathematical Problems in Engineering, vol. 2014, Article ID 792792, 9 pages, 2014.

[41] Z.-H. Hu, Y. Zhao, and T.-M. Choi, "Vehicle routing problem for fashion supply chains with cross-docking," Mathematical Problems in Engineering, vol. 2013, Article ID 362980, 10 pages, 2013.

[42] R. Hammami and Y. Frein, "Integration of the profit-split transfer pricing method in the design of global supply chains with a focus on offshoring context," Computers \& Industrial Engineering, vol. 76, pp. 243-252, 2014.

[43] G. Q. Huang and S. X. Xu, "Truthful multi-unit transportation procurement auctions for logistics e-marketplaces," Transportation Research B: Methodological, vol. 47, pp. 127-148, 2013.

[44] P. M. Reyes, "Logistics networks: a game theory application for solving the transshipment problem," Applied Mathematics and Computation, vol. 168, no. 2, pp. 1419-1431, 2005.

[45] L. S. Shapley, "A value for n-person Games," Annals of Mathematics Studies, vol. 28, pp. 307-317, 1953.

[46] P.-Y. Yin, S.-S. Yu, P.-P. Wang, and Y.-T. Wang, "A hybrid particle swarm optimization algorithm for optimal task assignment in distributed systems," Computer Standards and Interfaces, vol. 28, no. 4, pp. 441-450, 2006.

[47] T. J. Ai and V. Kachitvichyanukul, "Particle swarm optimization and two solution representations for solving the capacitated vehicle routing problem," Computers and Industrial Engineering, vol. 56, no. 1, pp. 380-387, 2009.

[48] Y. Marinakis and M. Marinaki, "A hybrid multi-swarm particle swarm optimization algorithm for the probabilistic traveling saleman problem," Computers and Operations Research, vol. 37, no. 3, pp. 432-442, 2010.

[49] R. J. Kuo and Y. S. Han, "A hybrid of genetic algorithm and particle swarm optimization for solving bi-level linear programming problem-a case study on supply chain model," Applied Mathematical Modelling, vol. 35, no. 8, pp. 3905-3917, 2011.

[50] B. F. Moghaddam, R. Ruiz, and S. J. Sadjadi, "Vehicle routing problem with uncertain demands: an advanced particle swarm algorithm," Computers and Industrial Engineering, vol. 62, no. 1, pp. 306-317, 2012. 
[51] K. Yang, Y. K. Liu, and G. Q. Yang, "An improved hybrid particle swarm optimization algorithm for fuzzy p-hub center problem," Computers \& Industrial Engineering, vol. 64, no. 1, pp. 133-142, 2013.

[52] Y. Wang, X. L. Ma, Y. T. Lao, Y. H. Wang, and H. J. Mao, "Vehicle routing problem: simultaneous deliveries and pickups with split loads and time windows," Transportation Research Record, vol. 2378, no. 1, pp. 120-128, 2013.

[53] Y. Wang, X.-L. Ma, Y.-T. Lao, H.-Y. Yu, and Y. Liu, "A twostage heuristic method for vehicle routing problem with split deliveries and pickups," Journal of Zhejiang University: Science C, vol. 15, no. 3, pp. 200-210, 2014.

[54] Y. Marinakis, M. Marinaki, and G. Dounias, "A hybrid particle swarm optimization algorithm for the vehicle routing problem," Engineering Applications of Artificial Intelligence, vol. 23, no. 4, pp. 463-472, 2010.

[55] F. P. Goksal, I. Karaoglan, and F. Altiparmak, "A hybrid discrete particle swarm optimization for vehicle routing problem with simultaneous pickup and delivery," Computers \& Industrial Engineering, vol. 65, no. 1, pp. 39-53, 2013. 


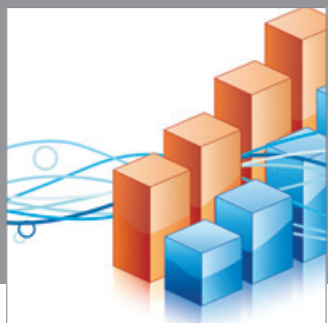

Advances in

Operations Research

mansans

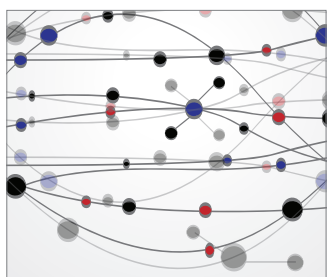

The Scientific World Journal
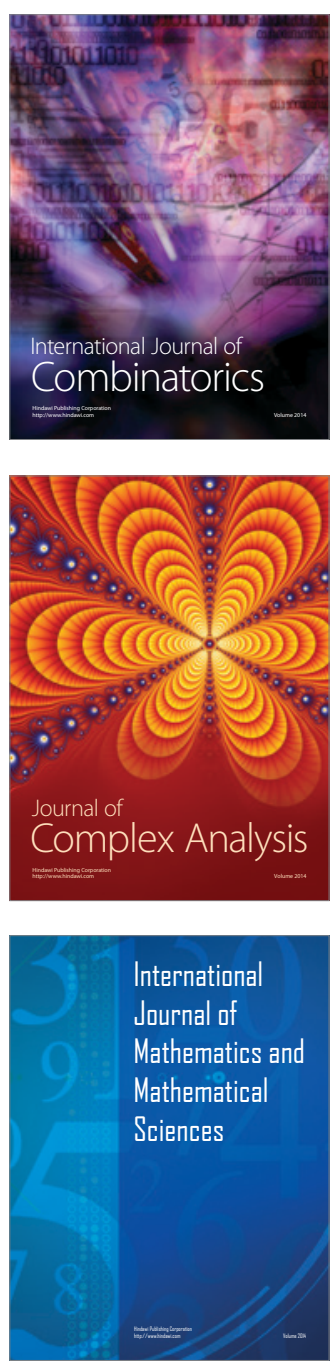
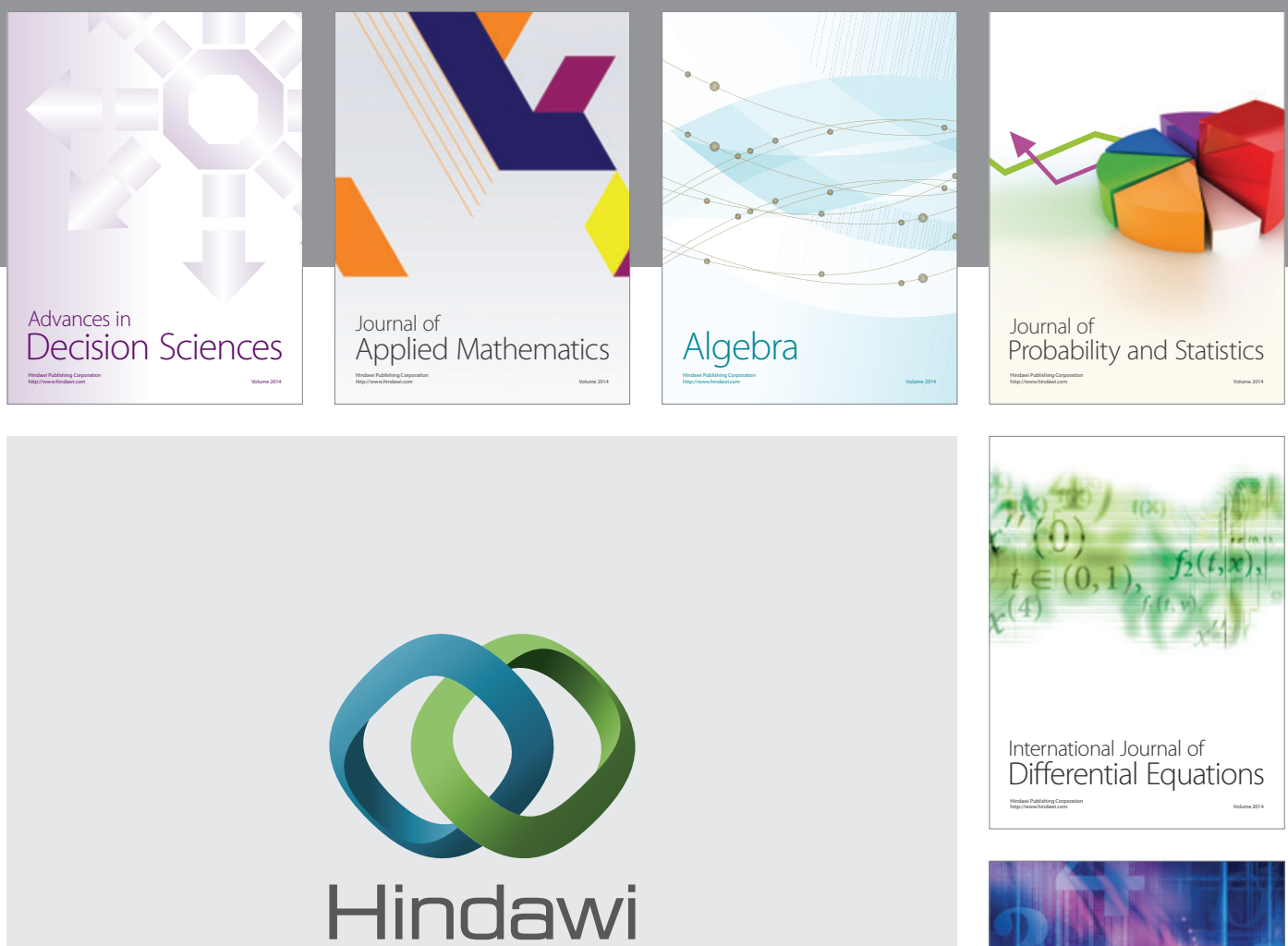

Submit your manuscripts at http://www.hindawi.com
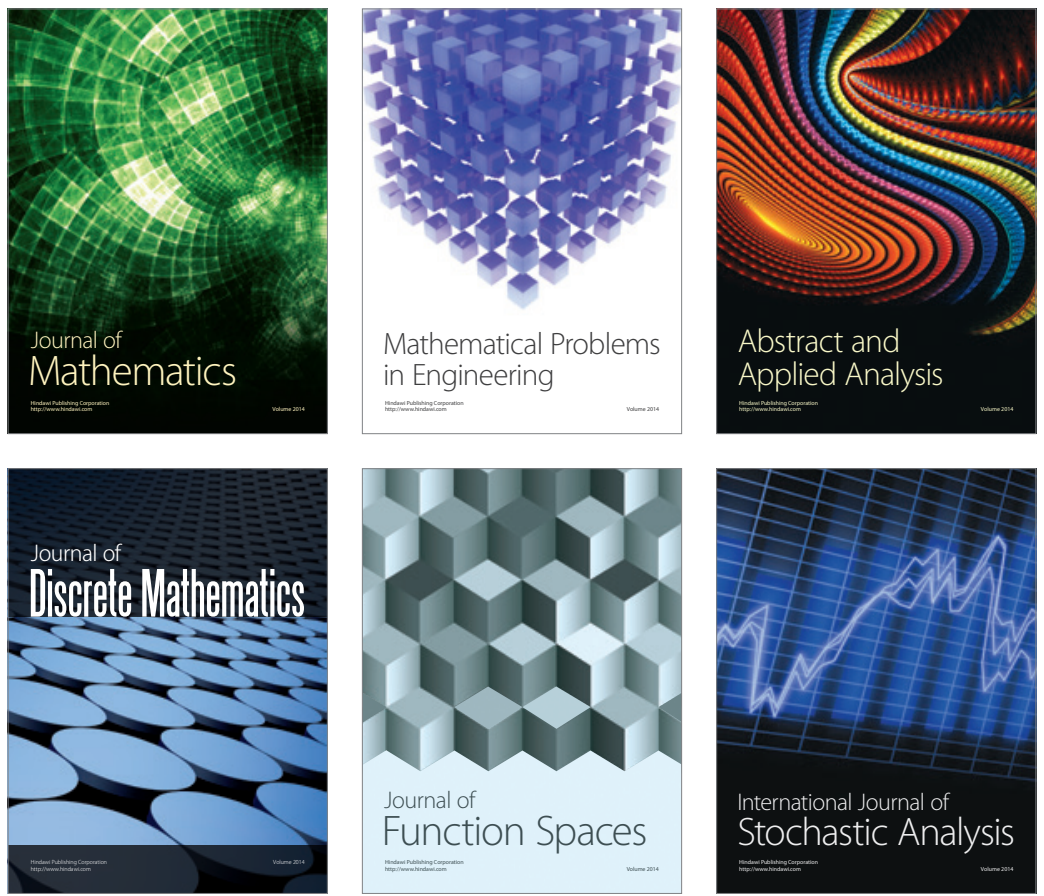

Journal of

Function Spaces

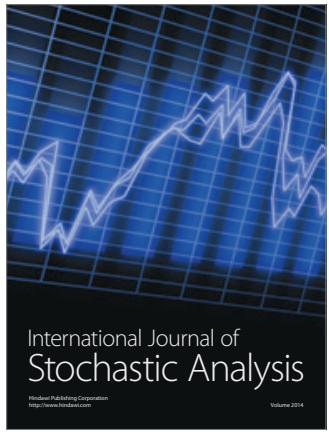

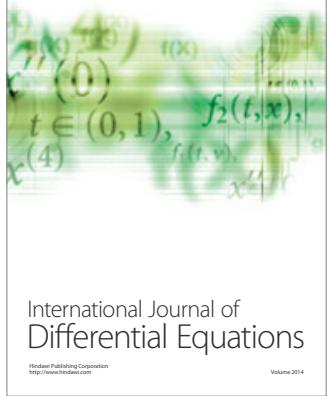
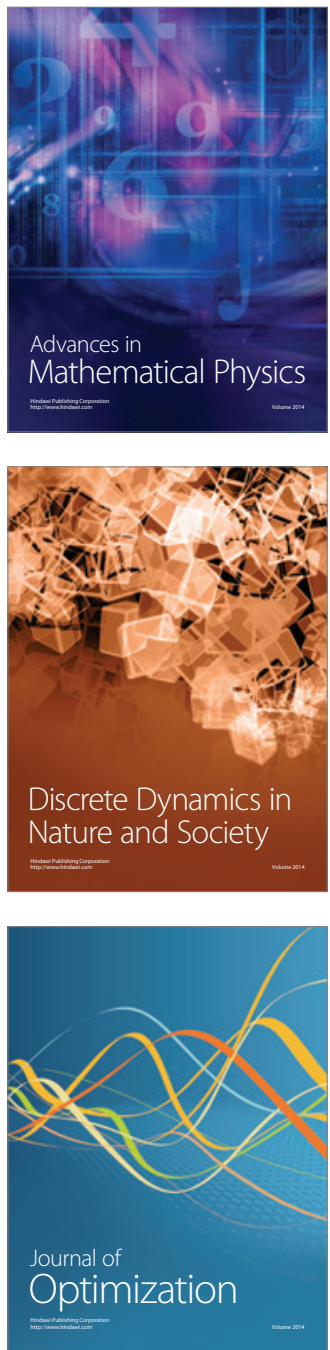